\title{
Fetale MRT-Diagnostik
}

\author{
Franz Wolfgang Hirsch, Ina Sorge
}

\section{Übersicht}

Einleitung

Praktische Durchführung

Sequenzen

Allgemeines zu Indikationen

Sicherheit

Zentrales Nervensystem

Thorax

Abdominelle und retroperitoneale Fragestellungen

\section{Einleitung}

Die fetale MRT-Diagnostik hat sich als bildgebende Ergänzungsmethode, insbesondere im Bereich der Hirndiagnostik etabliert [1]. Das MRT erbringt aufgrund der differenten Gewebeabbildung und des höheren Weichteilkontrasts eine zum Ultraschall (US) komplementäre Information.

In Praxen oder kleineren Krankenhäusern wird fetales MRT selten ausgeführt, da die Expertise hier aufgrund niedriger Fallzahlen und der notwendigen interdisziplinären Diskussion der MRT-Befunde schwerer zu erreichen ist. Für interdisziplinär ausgerichtete Fetalzentren ist jedoch die additive Mehrinformation des fetalen MRT für bestimmte Indikationen inzwischen unverzichtbar geworden.

Vor jeder fetalen MRT-Diagnostik muss ein qualifizierter Ultraschallbefund vorliegen, aus dem sich eine Indikation ableitet und eine Fragestellung an das fetale MRT ergibt. Die Indikationsbreite für das fetale MRT reicht dann von der Frage nach assoziierten Fehlbildungen bis hin zur Planung bei fetaler Chirurgie [2]. Auch für die Unterstützung des elterlichen Verständnisses für eine komplexe Pathologie und die nachfolgende interdisziplinäre Beratung kann ein MRT hilfreich sein.

\section{Tipp für die Praxis}

Bei einzelnen Krankheitsentitäten, z. B. der Myelomeningozele, hat sich das unmittelbar pränatal durchgeführte MRT als gleichwertig mit der postpartalen Diagnostik herausgestellt. In diesen Fällen ist die In-utero-Untersuchung schonender für das Kind.

Wegen der kindlichen Bewegungen und der damit notwendigen schnellen Sequenzen erreicht das fetale MRT bisher nicht die hohe räumliche Auflösung, die heute bei postnatalen Standard-MRT-Untersuchungen an Kindern erwartet werden kann (Abb. 1). Bei guten High-End-Ultraschallgeräten liegt die räumliche Auflösung über der räumlichen Auflösung von 1,5-TeslaMRT-Geräten. Bei 3,0-Tesla-Geräten ist sie etwa gleich gut. Im Vergleich dazu ist jedoch die Kontrastauflösung der MRT deutlich besser als im Ultraschall.

Durch die Komplementarität von Kontrast- und räumlicher Auflösung ergänzen sich MRT und Ultraschall ideal.

Der indikationsgerechte Einsatz des MRT als „SecondLine-Imaging“ ist heute Gegenstand wissenschaftlicher Studien. Im Schädelbereich hat sich die fetale MRTDiagnostik als Ergänzung fest etabliert [3]. Bei bestimmten Entitäten des Thorax- und Abdomenbereichs ist ein Zusatznutzen sehr wahrscheinlich. Bei seltene- 

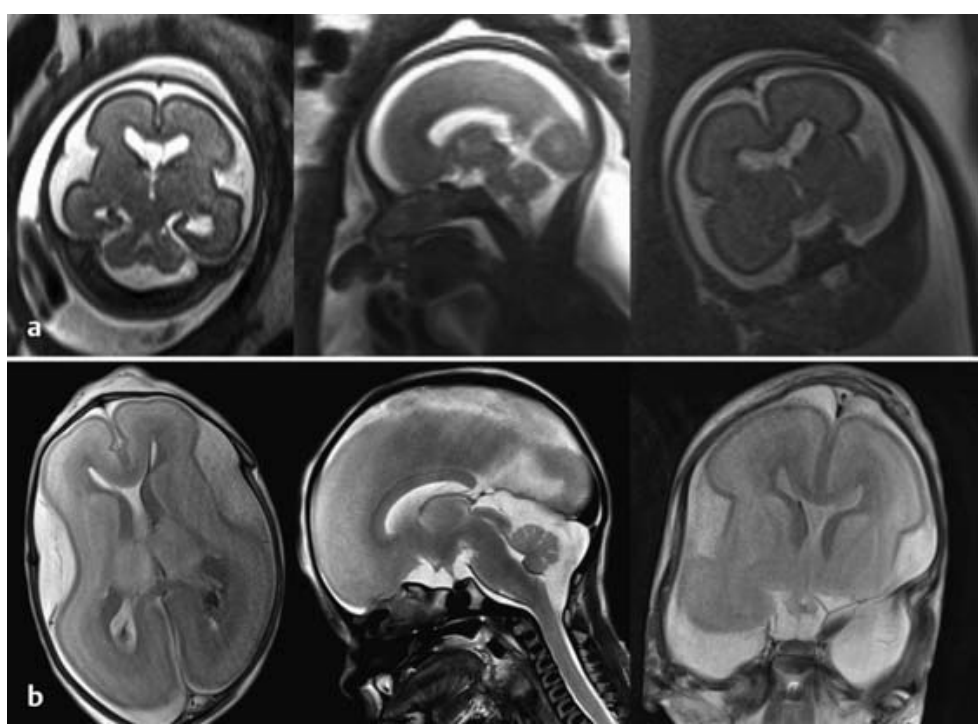

Abb. 1 a und b Vergleich eines pränatalen MRT in der 22. SSW (a: obere Reihe, Messdauer 28 Sekunden pro Sequenz) mit einem postmortalen MRT bei einem gleichaltrigen Feten (b: untere Reihe, Messdauer 8 Minuten pro Sequenz). Das pränatale MRT hat aufgrund der Kürze der Datenakquisition eine schlechtere räumliche Auflösung und einen niedrigeren Weichteilkontrast.

\section{Tipp für die Praxis}

Die Patientin wird mit den Füßen zuerst in das Gerät eingefahren, um klaustrophobische Reaktionen zu vermeiden.

Die Untersuchung beginnt mit 3 Übersichtssequenzen von $5 \mathrm{~mm}$ Schichtdicke, um die Lage des Kindes im Uterus zu orten. Erst die nachfolgenden Untersuchungssequenzen bringen dann die gewünschte diagnostische Information, wobei immer 3 exakt orthogonal zueinander stehende Schichtorientierungen angestrebt werden.

Wegen der Bewegungen des Kindes wird die jeweils nächste Untersuchungssequenz immer auf der letzten, zuvor beendeten MRT-Sequenz geplant. Dadurch wird gewährleistet, dass keine Fehlmessungen in falschen Kindspositionen entstehen. Die MRT-Untersuchung wird dadurch - analog zum Ultraschall - zu einer interaktiven Untersuchung. Die Durchführung einer guten fetalen MRT-Untersuchung wird dadurch allerdings auch untersucherabhängig und ist nur mit geschultem Assistenzpersonal durchführbar.

ren Anomalien ist die Datenlage noch zu unsicher kasuistisch, um über den diagnostischen Mehrgewinn des MRT eine Aussage treffen zu können [4].

Das fetale MRT weist gegenüber dem Ultraschall einen deutlich höheren Weichteilkontrast auf und ist daher methodenbedingt in der Gewebedifferenzierung überlegen.

\section{Praktische Durchführung}

Der Durchmesser der MRT-Geräteöffnung (Gantry) beträgt $60 \mathrm{~cm}$, bei einigen neueren Geräten auch $70 \mathrm{~cm}$. Dieses Gerätemaß kann sowohl bei adipösen Frauen als auch in der späten Schwangerschaft limitierend sein. Das Gleiche trifft für Zwillingsschwangerschaften zu.

Die Patientinnen werden bis zur 29. SSW in Rückenposition gelagert, ab der 30. SSW in der Regel in Seitenlage.

Auf das Abdomen der Patientin wird eine lokale Spule (eine sogenannte Body-Array-Spule) in den Abmessungen $50 \times 60 \times 5 \mathrm{~cm}$ aufgelegt und mit Gurten befestigt. Diese lokale Spule empfängt das Nutzsignal vom Fetus. Das umgebende MRT-Gerät stellt dagegen nur das homogene Magnetfeld (1,5 Tesla oder 3,0 Tesla) und die sog. Gradientenfelder zur Verfügung.
In $90 \%$ der MRT-Untersuchungen ist mit einer guten Bildqualität zu rechnen, in $10 \%$ der Fälle kommt es durch Kindsbewegungen zu einer eingeschränkten Beurteilbarkeit.

Eine einzelne Sequenz mit ca. 20 Bildern dauert im Durchschnitt 20-30 Sekunden. Zusammen mit den Abstimmzeiten zwischen den Sequenzen (Shim-Vorgang) und der Notwendigkeit, einige Sequenzen bei Bewegungsunschärfen zu wiederholen, dauert eine fetale MRT-Untersuchung 20-30 Minuten. Bei Zwillingsschwangerschaften oder sehr diffizilen Fragestellungen kann sich die Untersuchungszeit bis auf 30-45 Minuten verlängern.

Adipositas und Oligohydramnion beeinflussen die Bildqualität nicht negativ.

\section{Sequenzen}

Obwohl die MRT-Diagnostik seit 25 Jahren etabliert ist, konnte sich die fetale MRT erst mit der Einführung von ultraschnellen T2-Sequenzen seit 10 Jahren in der Fetaldiagnostik etablieren. Die Akquisition von ca. 20 Bildern in 20 Sekunden wird mit dem Nachteil einer deutlich reduzierten örtlichen Auflösung erkauft. 
Dadurch lassen sich die prinzipiellen Vorteile der MRT-Diagnostik im Fetalbereich bisher nicht vollständig realisieren. Die einzelne Schichtdicke beträgt im Regelfall 3 mm. Dünnere Schichtdicken sind möglich, bringen aber verrauschtere Bilder.

Ultraschnelle T2-gewichtete Sequenzen. Sie sind das „Arbeitspferd“ der fetalen MRT-Diagnostik. Eine alleinige T2-Bildgebung ist heute aber nicht mehr ausreichend.

T2-Dickschichtbilder können hilfreich bei der Darstellung von ausgeprägten Pathologien sein, die auf einem Übersichtsbild visualisiert werden sollen. Da sie mit einer Frequenz von 10 Bildern/s zu erhalten sind, dienen sie zudem als dynamische Sequenzen zur Visualisierung von kindlichen Bewegungen und internen fetalen Bewegungen (Zwerchfellbewegungen etc.).

T2-Standard-Bilder werden entweder als T2-HASTEoder als T2-SSFP-Bilder ausgeführt. Beide Methoden haben Vor- und Nachteile, doch es gibt auch gerätetechnische Unterschiede und Qualitätsunterschiede, sodass die Entscheidung zwischen beiden Sequenztypen gemeinsam vor Ort mit dem Radiologen getroffen werden sollte. SSFP-Bilder sind an neueren 3,0-Tesla-Geräten den T2-HASTE-Bildern überlegen.

T1-gewichtete Sequenzen. Diese werden benötigt, um den Darm, die Leber und die Schilddrüse sowie die Hypophyse abzubilden. Sie dienen darüber hinaus dem spezifischen Blutnachweis (Methämoglobin), insbesondere im Gehirn.

Ultraschnelle Sequenzen in T2-Wichtungen in allen 3 Ebenen und eine Ebene einer schnellen T1-Wichtung gehören zum Basisprotokoll einer fetalen MRT-Untersuchung.

Weitere Wichtungen. Bei speziellen Fragestellungen sind weitere Wichtungen notwendig: Dazu gehören Diffusionswichtungen mit einem b-Wert von 700 zur Visualisierung von Infarkten und zur Nierendarstellung bei Verdacht auf Gewebeanomalien. Diffusionswichtungen werden darüber hinaus auch zur Faserverlaufsdarstellung des Gehirns verwendet. Diese Untersuchungen befinden sich aber noch in einem experimentellen Stadium (Abb. 2) [5]. Gleiches gilt für die Spektroskopie: Wegen der langen Sequenzdauer von über 4 Minuten sind verlässliche Informationen über Stoffwechselprodukte des fetalen Hirnes ohne kindliche Sedierung bisher nicht routinemäßig zu gewinnen.

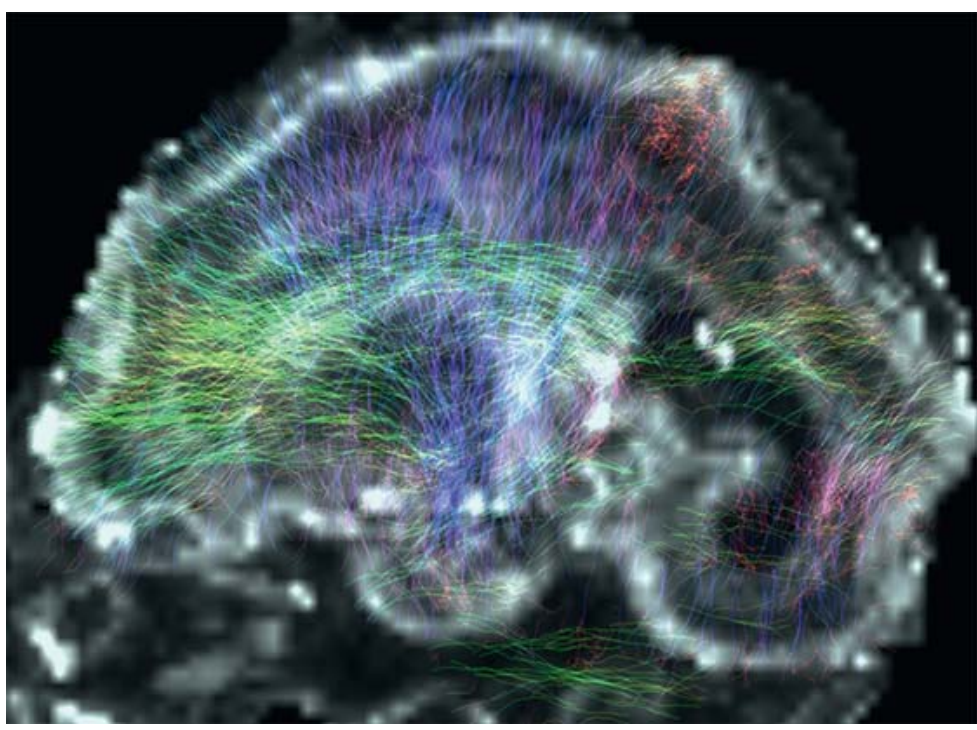

Abb. 2 Faserdarstellung (Traktografie) mittels Diffusionsbildgebung in einem postmortalen MRT eines Fetus in der 22. SSW. Unterschiedliche Faserverläufe werden mit unterschiedlichen Farben dargestellt. Der Verlauf der Pyramidenbahn (blau) ist auch in der 22. SSW schon gut zu erkennen.

Akzeptierte fetale MRT-Fragestellungen $[7,8]$

- Adipositas per magna

- ausgeprägtes Oligohydramnion

- komplexe Hirnfehlbildungen, Hirntumoren, Hirnblutungen

- Lungenfehlbildungen und Zwerchfelldefekte (Lungenvolumina und Lungenreife)

- Darmatresie

- Bauchwanddefekte

- fetale Tumoren

- Vermeidung einer erneuten postnatalen Bildgebung vor geplanter OP (z. B. Myelomeningozele) - Mutter als „schonender“ Transportinkubator

- übersichtliche Visualisierung bei älteren Feten für den nachbehandelnden Kinderchirurgen (z. B. bei Omphalozele)

- unklare Ultraschallbefunde trotz qualifizierter US-Untersuchung (DEGUM II und III) vor einem interdisziplinären Konsil

\section{Allgemeines zu Indikationen}

Gründe für den Einsatz der fetalen MRT-Diagnostik sind mütterlicher oder kindlicher Natur.

Mütterliche Indikationen. In Situationen, in denen die Ultraschalluntersuchung eine unzureichende Information ergibt (schlechte Schallbedingungen, z. B. durch ausgeprägte Adipositas oder Oligohydramnion oder Hirndiagnostik bei schon tief im Becken stehendem Kopf), ist die MRT-Diagnostik eine Alternative zum Ultraschall. 
Kindliche Indikationen. Aufgrund des besonders guten Weichteilkontrasts ist die MRT für den Ausschluss oder die Bestätigung von zerebralen Anomalien und für die Darstellung von komplexen Pathologien im Hirnbereich hilfreich [6].

Raumforderungen im Hals-Thorax-Bereich mit Trachealeinengung, Lungenfehlbildungen und Zwerchfellhernien sind die häufigsten Untersuchungsindikationen im Thoraxbereich.

Im Abdomen stehen Tumoren (Teratome oder Lebertumoren) sowie die Darmobstruktionen bezüglich der Indikationsstellungen im Vordergrund. Insbesondere die signalreiche Darstellung des Mekoniums erleichtert die Darmbeurteilung des Feten im MRT. Bei Bauchwanddefekten wird von Kinderchirurgen häufig eine MRT-Visualisierung gewünscht. Details zu Indikationen sind den einzelnen Organsystemen zugeordnet.

Kontraindikationen. Magnetisches Metall im Körper kann das Magnetfeld stören und damit lokal die Beurteilung behindern. Äußere Metallgegenstände wie Gürtel mit Metallverschlüssen, auch BH und lokales Piercing sind vor der Untersuchung zu entfernen. Medizinisch eingebrachtes Metallmaterial besteht in der Regel aus Titan, dieses ist amagnetisch und daher keine Kontraindikation.

Tipp für die Praxis

Cave! Bei komplexen implantierten Geräten (CochleaImplantat, Pacemaker, Baclofen-Pumpe etc.) muss stets ein Gerätepass vorgelegt werden, der die MRT-Tauglichkeit explizit bestätigt. Dieses sollte der Patientin vom überweisenden Gynäkologen im Vorfeld mitgeteilt werden.

\section{Sicherheit}

Bisher sind keine Langzeiteffekte durch MRT-Untersuchungen an Mutter oder Fetus beschrieben worden. Als potenzielle Gefahr werden aber folgende Punkte diskutiert:

- Energieeintrag. Der Energieeintrag in Mutter und Fetus durch das statische und das Gradienten-Magnetfeld wird bei jeder einzelnen Untersuchung als spezifische Energieabsorptionsrate (SAR) gemessen und berechnet. Je nach Land legt eine Kommission die zulässige Höhe des SAR-Limits mit einem entsprechenden Sicherheitsfaktor fest. Eine Überschreitung von diesen Grenzwerten ist geräteseitig aus- geschlossen und nur im Rahmen von genehmigten Studien möglich. Die maximale Temperaturerhöhung der Patientin muss weniger als $0,5^{\circ} \mathrm{C}$ betragen. In Tierexperimenten fand sich bisher kein Nachweis von teratogenen Effekten oder chromosomalen Veränderungen. Obwohl in 20 Jahren seit der breiten Einführung der MRT-Diagnostik keine Nebeneffekte gesehen wurden, empfiehlt das National Radiation Protection Board (USA) die Durchführung von fetalen MRT-Untersuchungen erst ab dem Beginn des 2. Trimenons, obwohl es dafür keine wissenschaftliche Evidenz gibt.

- Akustische Belästigung des Fetus. Die hohe Lautstärke von bis zu 98 dB im MRT-Gerät erreicht den Fetus nicht. Die Dämpfung durch das Fruchtwasser führt zu Schalldrücken von bis zu 30 dB am Fetus. Eine ursächliche Hörschädigung nach fetaler MRT-Diagnostik wurde bisher nicht berichtet.

- Kontrastmittelapplikation. Es gibt widersprüchliche Angaben über die Durchgängigkeit der Plazentaschranke für Gadolinium. Da aber eine gewisse Menge Gadolinium-Chelat die Plazenta passiert und die Gefahr von Ablagerungen oder toxischen Schäden durch freies Gadolinium nicht auszuschließen ist, wird der Gebrauch von Kontrastmittel in der Schwangerschaft nicht empfohlen.

Bisher gibt es keinen Nachweis einer Schädigung des Feten durch die Kernspintomografie.

\section{Zentrales Nervensystem}

Untersuchungen des zentralen Nervensystems sind die häufigste Indikation für eine fetale MRT-Diagnostik [9].

Vorteile der fetalen MRT-Diagnostik sind:

- Deutlich besserer Weichteilkontrast der MRT-Bilder im Vergleich zum Ultraschall. Dieser ermöglicht eine bessere Differenzierung zwischen weißer und grauer Substanz. Es sind mit der MRT zudem die streng regelhaft ablaufende Myelinisierung, die neuronale Migration und die Gyrierung sowie deren Störungen erkennbar.

- Keine Beeinträchtigung der Bildgebung durch die zunehmende Kalzifizierung der Kalotte.

- Orthogonale Darstellung von 3 exakt aufeinander senkrecht stehenden Ebenen.

- Im Vergleich zum US klarere Darstellung der hinteren Schädelgrube und des kraniozervikalen Übergangs. 
- Bessere Darstellung von ischämischen, hämorrhagischen und entzündlichen Läsionen.

- Eine präzise morphologische Diagnostik und die interdisziplinäre Beratung der Eltern bezüglich der sich daraus ergebenden klinischen Konsequenzen sind für die Entscheidung der Eltern für oder gegen einen Schwangerschaftsabbruch von zentraler Wichtigkeit [10]. Es besteht Konsens, dass durch das fetale MRT des Hirnes in entscheidungsrelevanten Situationen neue Aspekte zum US hinzugefügt werden können. So erfordert insbesondere die Einschätzung der neuronalen Migration, der Nachweis von Heterotopien und die genaue Fehlbildungsklassifikation eine ergänzende MRT-Diagnostik.

\section{Hirnentwicklung}

Die neuronale Migration und die Hirnfaltung verlaufen nach streng zeitlich festgelegten Abläufen, deren Varianz nur gering ist. Der Nachweis der 4 Schichtungen des Großhirns vor der 28. SSW und deren zeitgerechtes Verschwinden danach ist ein wichtiger Marker für eine ungestörte Hirnentwicklung. Im MRT sind vor der 28. SSW folgende Zonen von innen nach außen erkennbar:

- Ventrikulärzone

- intermediäre Zone

- Subplate

- Cortical Plate (Abb. 3 und 4) [11]

Das Ausbleiben dieser phasenhaften Entwicklung und die Verzögerung der sich in engen zeitlichen Grenzen vollziehenden Gyrierung weist auf eine zerebrale Fehlentwicklung hin. Folge können dann schwerwiegende Erkrankungen wie Heterotopien, Lissenzephalie und andere Gyrierungsstörungen sein [12]

Die neuronale Migration ist bildgebend ausschließlich im MRT zu erkennen.

\section{Ventrikulomegalie und Hydrozephalus}

Moderat erweiterte Ventrikel sind häufig. Bei einer konstanten Borderline-Ventrikelweite von 10-12 mm ist eine MRT-Diagnostik nicht notwendig, da das Outcome in diesen Fällen ohnehin gut ist. Wegen der schlechten Prognose sollten aber schwere Hydrozephali mit einer Ventrikelweite über $20 \mathrm{~mm}$ stets im MRT untersucht werden, da der Nachweis von assoziierten Fehlbildungen mit der MRT häufiger gelingt [13]. Typische Begleitfehlbildungen wie Schizenzephalie,
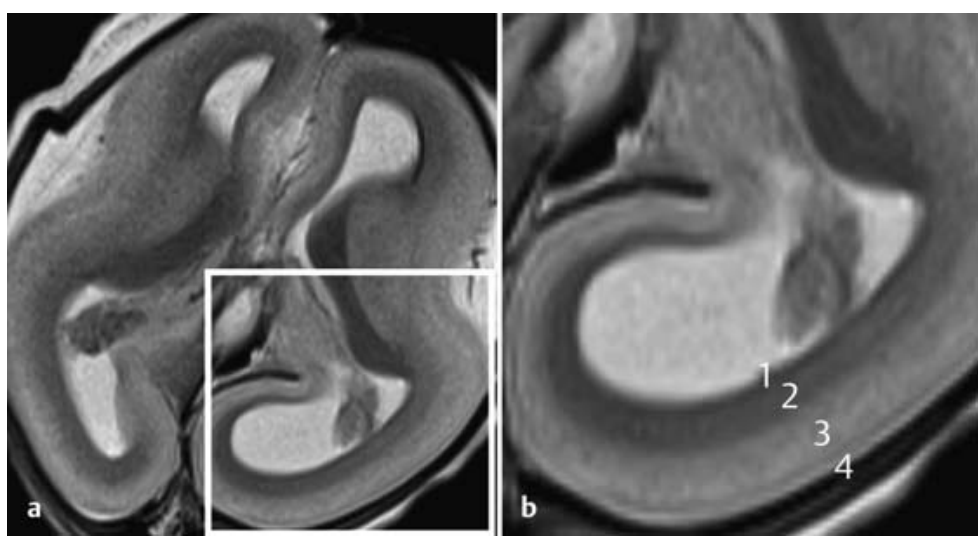

Abb. 3 a und b Normaler, zonaler Aufbau des Hirnmantels in der 20. SSW. a In dieser Schwangerschaftswoche ist der 4-lagig gegliederte Aufbau der Hirnstruktur noch nachweisbar, da die neuronale Migration der Nervenzellen aus der Ventrikulärzone in den Kortex noch nicht abgeschlossen ist. b Ausschnittvergrößerung: von innen nach außen (1) Ventrikulärzone, (2) Intermediärzone, (3) Subplate, (4) Cortical Plate.

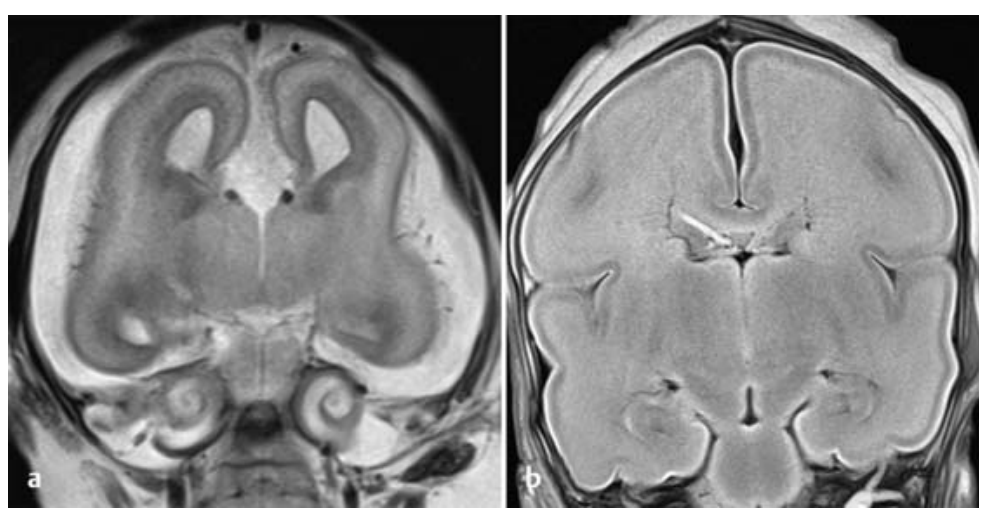

Abb. 4a und b Vergleich der Gyrierung und der Hirnschichtung in der 18. SSW (a) und der 28. SSW (b). In der 18. SSW ist die Hirnoberfläche noch glatt und der zonale Aufbau des Hirnes noch klar erkennbar. In der 28. SSW ist die Mehrzahl der Sulci und Gyri ausgebildet.

aber auch gering ausgebildete Fehlbildungen der hinteren Schädelgrube entziehen sich gelegentlich dem sonografischen Nachweis [14].

Mit dem MRT ist häufig die Ursache des Hydrozephalus zu diagnostizieren.

So ist z. B. ein X-linked-Hydrozephalus durch Aquäduktstenose an der Aquäduktkonfiguration und dem kleinen 4. Ventrikel erkennbar (Abb. 5).

\section{Fehlbildungen des zentralen Nervensystems}

\section{Fehlbildungen des Großhirns}

Balkenagenesie. Sie ist die häufigste Fehlbildung des Großhirns und sonografisch meist sicher an der Kolpozephalie - den unförmigen erweiterten Hinterhörnern bei schmalen Vorderhörnern - zu erkennen. Im MRT 

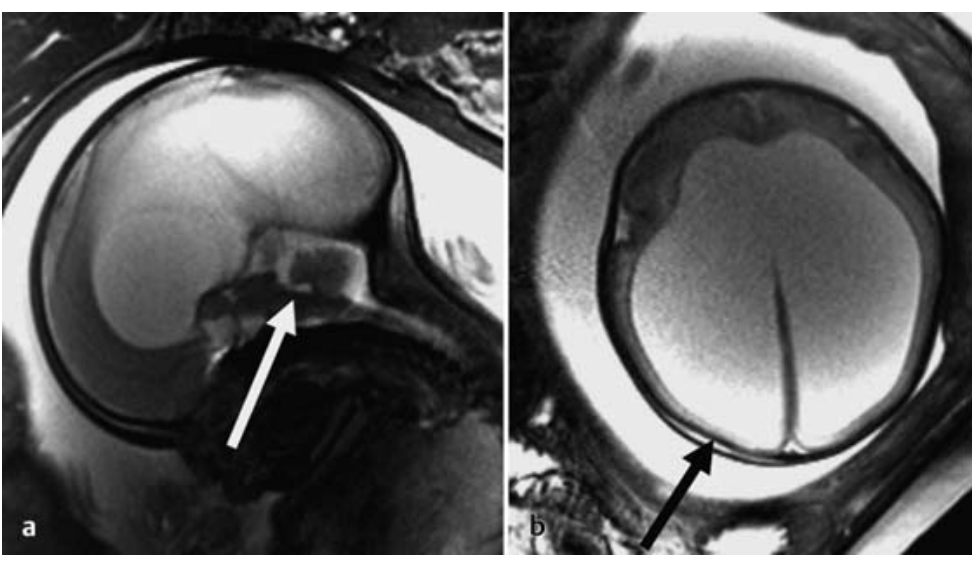

Abb. 5 a und b Aquäduktstenose, 24. SSW bei später genetisch bestätigtem x-linked Hydrozephalus. a Kleiner 4. Ventrikel und $\mathbf{b}$ massive Erweiterung der Seitenventrikel mit deutlicher Verschmälerung des Hirnmantels.
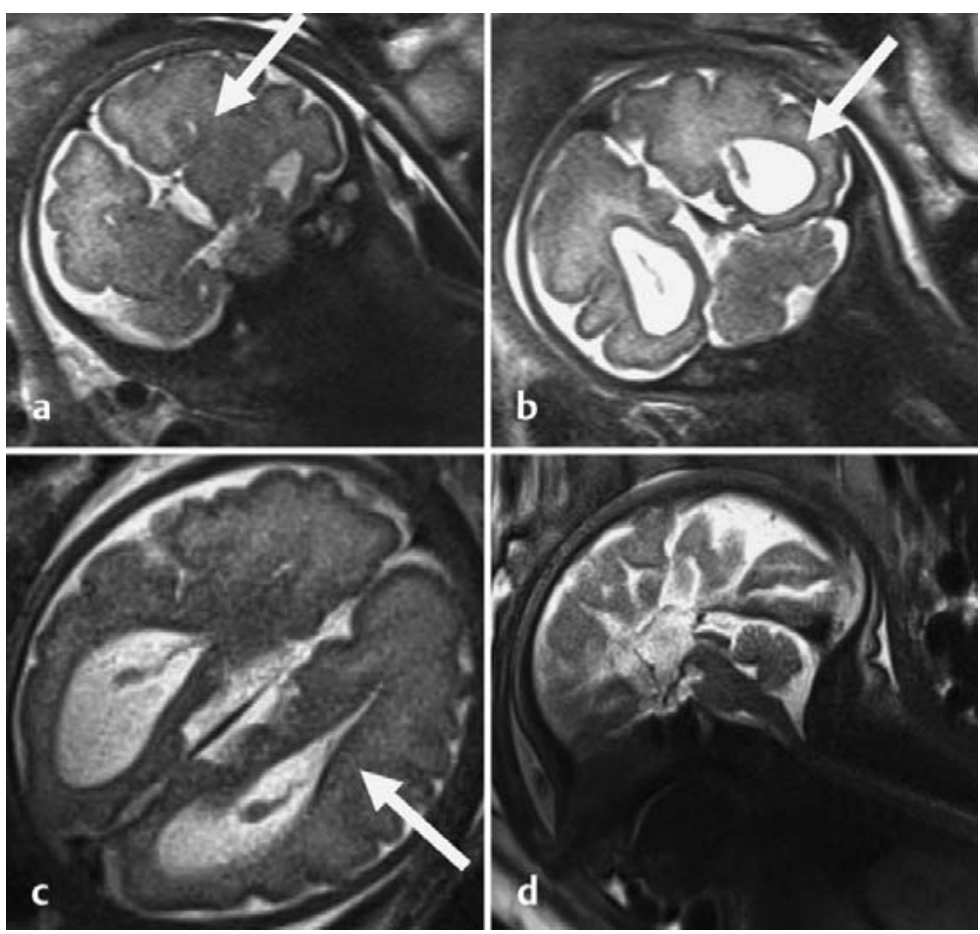

Abb. 6a bis d Balkenagenesie, 31. SSW. a Typische Stierhornform der Ventrikel, b Erweiterung der Hinterhörner der Seitenventrikel (Kolpozephalie), c Parallelverlauf der Seitenventrikel, d typisches Hahnenkammbild im Sagittalschnitt - die Sulci reichen bis unmittelbar an den 3. Ventrikel heran.
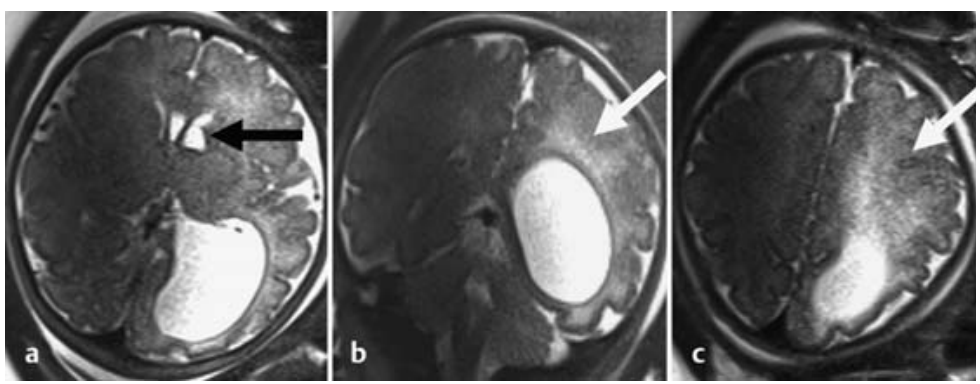

Abb. 7a bis c Überweisung wegen unklarem Hydrozephalus in der 34. SSW. Das MRT zeigt die einseitige Ventrikelerweiterung. Ursache ist eine Blockade des linken Foramen Monroi durch eine neuroepitheliale Zyste (schwarzer Pfeil, a). Das hellere Signal des linksseitigen Marklagers spricht für eine druckbedingte Liquordiapedese (weiße Pfeile, b, c). lässt sich die Balkenstruktur oder ihr Fehlen in sagittalen und koronaren Schnitten gut identifizieren. Beim Fehlen des Balkens finden sich unmittelbar an den 3. Ventrikel reichende Gyri (sog. „Hahnenkammzeichen“). Typisch ist die Stierhornform der Seitenventrikelvorderhörner im Koronarschnitt und der Parallelverlauf der Seitenventrikel im Transversalschnitt (Abb. 6). Die wichtigste Fragestellung an das MRT ist der Nachweis bzw. Ausschluss von Hirnfehlbildungen, da diese die Prognose eines Balkenmangels entscheidend beeinflussen.

Zysten. Diese sind häufig und können klinisch sehr relevant sein. Auch kleine Zysten können bei einer Lokalisation im Bereich der Liquorwege zu einer Abflussbehinderung, z. B. zu einer Blockade des Foramen Monroi, führen und damit einen einseitigen Hydrozephalus hervorrufen. Interhemisphärische Zysten sind stets ein Indikator für weitere Begleitfehlbildungen. Dagegen sind Arachnoidalzysten oft große, eindrucksvolle Befunde, allerdings klinisch meistens ohne Relevanz (Abb. 7-9).

Heterotopien der grauen Substanz. Hierbei handelt es sich um kongenitale Anomalien, die zu einer Epilepsie oder verzögerten geistigen Entwicklung führen können. Sie bestehen aus Zellnestern an grauer Substanz, die auf dem Weg der neuronalen Migration von der subependymalen germinalen Matrixzone zur Kortexoberfläche liegen geblieben sind.

Häufig gehen Heterotopien mit anderen Störungen der Kortexentwicklung einher, wie z.B. Polymikrogyrien oder kortikalen Dysplasien. Aber auch eine diffuse Heterotopie mit Pachygyrie bzw. Lissenzephalie ist möglich. Treten sie hingegen fokal isoliert auf, so können die Symptome später auch mild bzw. die Patienten asymptomatisch sein. Heterotopien können in verschiedenen Erscheinungsformen auftreten:

- Fokale Heterotopien. Dies sind noduläre Neuronenansammlungen, entweder entlang der Wand der Seitenventrikel, wobei der Kortex zumeist unauffällig oder verdünnt erscheint (Abb. 10), oder fokal innerhalb des Marklagers gelegen.

- Subkortikale bandförmige Heterotopien mit Gyrierungsstörungen. Sie sind deutlich seltener, jedoch häufiger mit kontralateralen Pyramidenbahnzeichen und fokal-motorischen Anfällen assoziiert. Die MRT zeigt typischerweise 2 parallele Schichten des Kortex (sog. Double Cortex Syndrome), bestehend aus einem dünneren inneren Band und einem dickeren äußeren Band, welche durch eine dünne Schicht weißer Substanz getrennt werden. 
- Diffuse Marklagerheterotopie. Diese ist immer verbunden mit einer Lissenzephalie (griech. = „glattes Gehirn“, Abb. 11). Die Lissenzephalie - als Überbegriff und gleichzeitig Maximalform einer Faltungsanomalie des Gehirns - ist durch einen Verlust der normalen Gyri und Sulci des Gehirns gekennzeichnet. Die Ausprägung dieser Malformation reicht dabei von einer Agyrie über die Pachygyrie bis hin zur nur lokalen Polymikrogyrie. Patienten mit einer Lissenzephalie haben typischerweise eine schwere geistige Behinderung, eine Epilepsie und häufig auch eine Mikrozephalie. In der MRT zeigt sich eine sanduhrglasförmige Konfiguration des Gehirns mit gelegentlich nur lokalen Anteilen von Pachygyrie oder Polymikrogyrie sowie einer flachen Sylvischen Fissur (Abb. 12) [15].

\section{Tipp für die Praxis}

Die Gyrierung entwickelt sich zwischen der 20. und 30. Schwangerschaftswoche und ist mit der MRT besser zu objektivieren als im Ultraschall. Die sichere Diagnose einer Gyrierungsstörung ist jedoch erst ab der 30. SSW möglich.

Schizenzephalie. Sie scheint im MRT sicherer diagnostizierbar zu sein als im Ultraschall, insbesondere bei offenen Spalten. Im MRT findet sich dann als zusätzliches Zeichen die signalarme graue Rindensubstanz entlang des Spaltes. Sie lässt sich vom Ventrikel bis zur Hirnoberfläche verfolgen (Abb. 13). Bei der geschlossenen Form der Schizenzephalie kann dieser Nachweis auch dem MRT entgehen, und man erkennt die Spaltbildung dann nur an einer lokalen Ausziehung der Ventrikelwand. Die graue Substanz zeigt im Bereich der Spalte und im angrenzenden Rindenbereich häufig eine Polymikrogyrie der Hirnoberfläche.

Fusionsstörungen. Diese kommen überwiegend innerhalb des Spektrums der Holoprosenzephalie vor. Während die ausgeprägten Formen (alobare Holoprosenzephalie, Abb. 14) leicht zu erkennen und meist nicht lebensfähig sind, sind Minimalformen (fehlendes Septum pellucidum als Ausdruck einer lobaren Form der Holoprosenzephalie) klinisch irrelevant. Bei jedem Verdacht auf eine Fusionsstörung muss insbesondere nach einer begleitenden Balkenanlagestörung, dem Vorhandensein der Sehnerven (septooptische Dysplasie) und einer Lippen-Kiefer-Gaumen-Spalte gesucht werden.

Meningozele und Enzephalozele. Im Bereich des Kopfes sind Meningozele und Enzephalozele durch den hohen Weichteilkontrast zwischen Gewebe und Liquor im
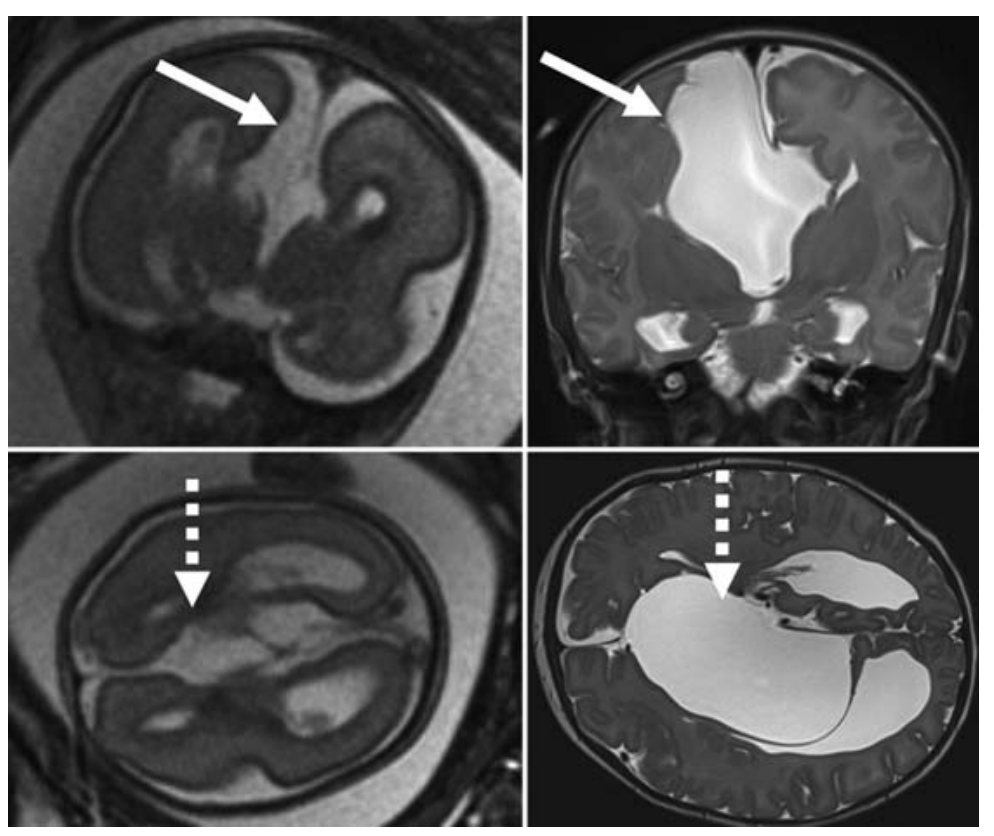

Abb. 8a und b Interhemisphärische Zyste Typ II bei Balkenagenesie. Links pränatale Bilder in der 20. SSW, rechts postnatales MRT im Alter von 3 Monaten. Die unmittelbare Zystenwand ist im MRT häufig nicht nachweisbar, dagegen die lokale Erweiterung im Interhemisphärenspalt.

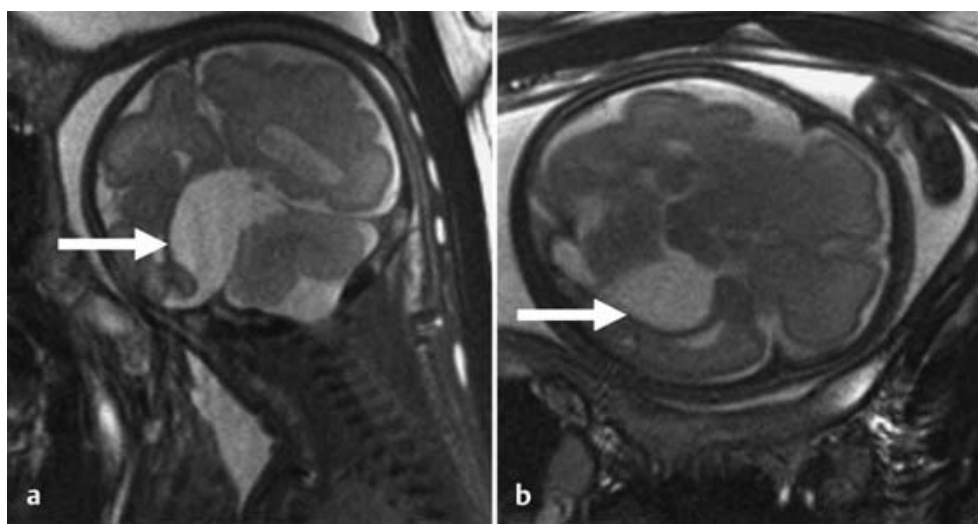

Abb. 9a und b Arachnoidalzyste rechts basal. Das Parenchym und der rechte Seitenventrikel werden durch die Zyste komprimiert.
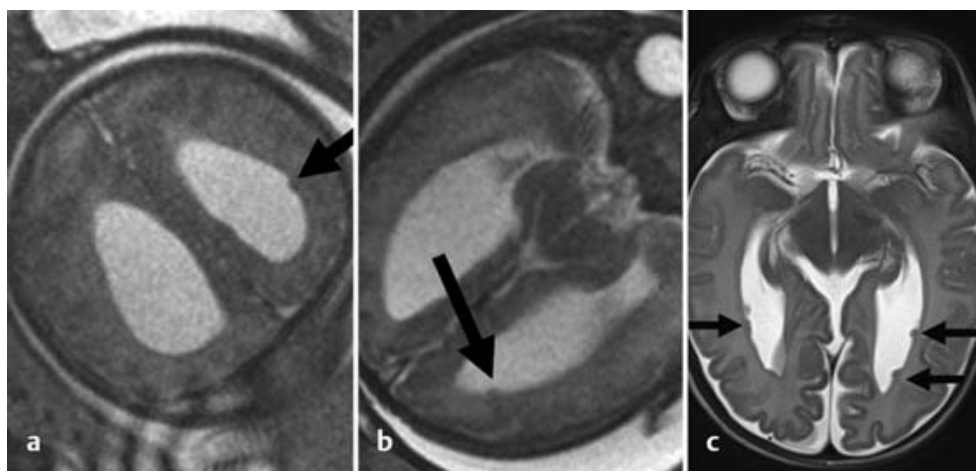

Abb. 10a bis c Fokale subependymale Heterotopien in der Wand der beiden Seitenventrikel. Die Heterotopien sind als kleine signalarme Vorwölbung in das Ventrikellumen zu erkennen (Pfeile in a und b; fetales MRT in der 32. SSW), c postnatales MRT im Alter von 8 Wochen. 


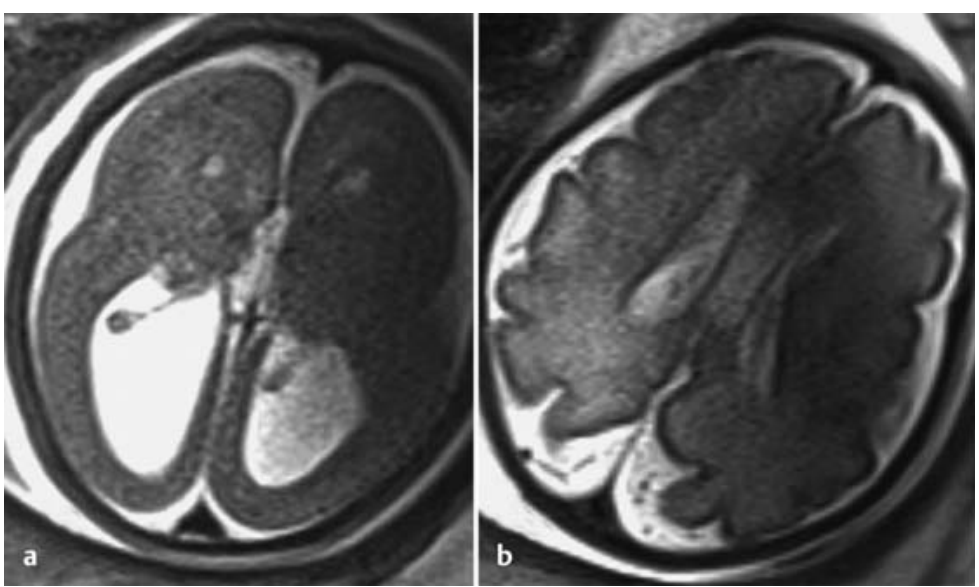

Abb. 11 a und b a Diffuse Marklagerheterotopie sowie altersuntypische glatte Hirnoberfläche (Lissenzephalie) bei einem Fetus der 33. SSW. Teilabbildung b zeigt dagegen die Hirnstruktur bei einem gleichaltrigen Fetus (33. SSW) zum Vergleich.
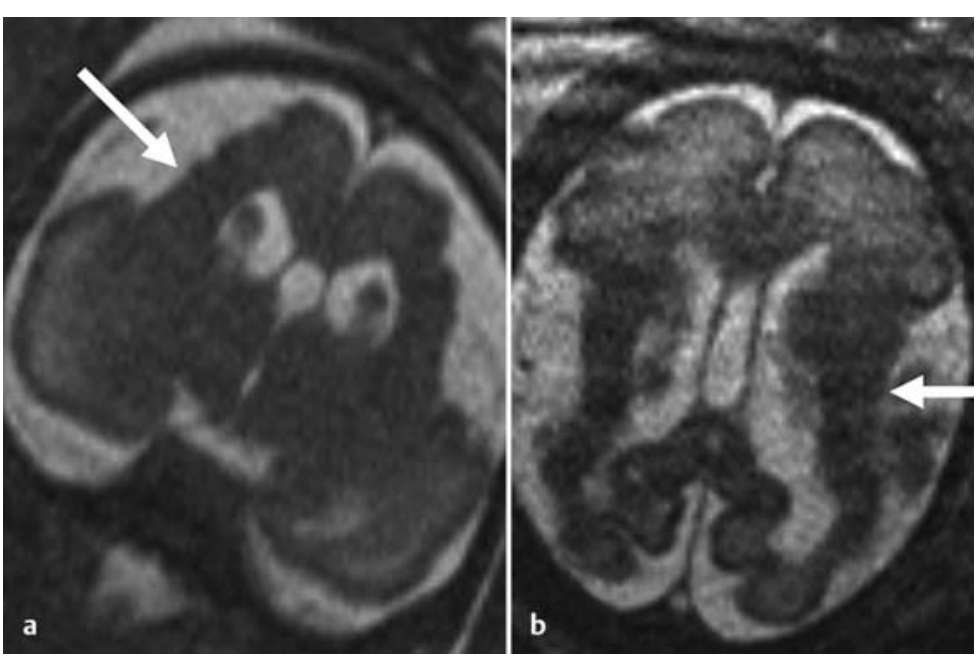

Abb. 12a und $\mathbf{b}$ Ausgeprägte lokale Gyrierungsstörung (Polymikrogyrie) bei einem Feten der 30. SSW. a Der Koronarschnitt zeigt eine Verschmälerung des parietalen Marklagers und die Polymikrogyrie, insbesondere im Bereich der Insula/Fissura Sylvii. b Transversalschnitt. Das Marklager enthält diffus verteilte heterotope graue Substanz und erscheint deshalb dunkel, Sanduhrglasform des Hirnes.

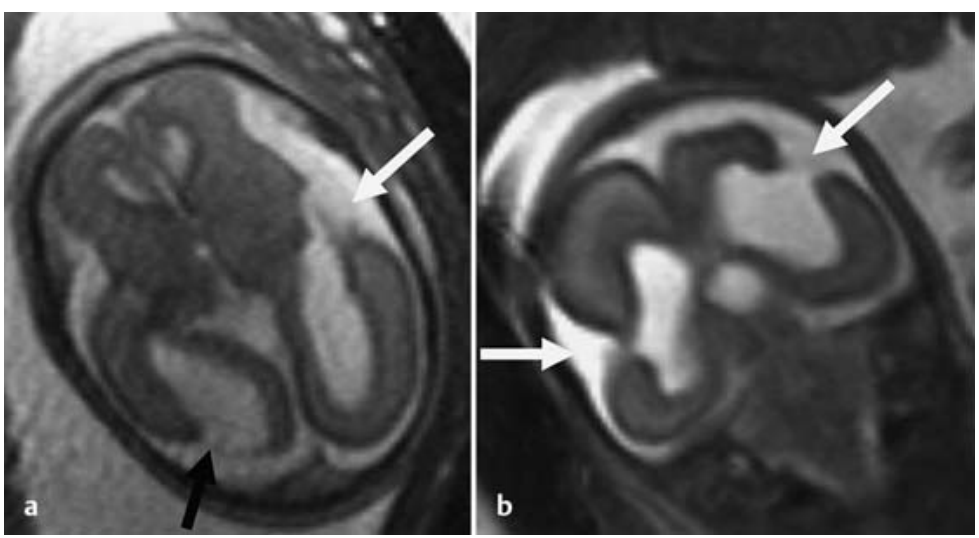

Abb. 13a und b Bilaterale offene Schizenzephalie: offene Spalten des Hirnmantels bei einem 20 Wochen alten Feten. Im Unterschied zu multiplen Infarkten reicht die graue Hirnsubstanz bei der Schizenzephalie bis an die Ventrikelwand heran (Pfeile).
MRT leicht zu diagnostizieren (Abb. 15-17). Meningozelen sind gegenüber den ebenso häufigen dysontogenetischen Zysten in der Mittellinie durch den Nachweis eines Knochendefekts abzugrenzen.

\section{- Kleinhirnfehlbildungen und Myelomeningozele}

Bei einem fetal diagnostizierten Hydrozephalus unklarer Genese ist stets ein dezidierter Ausschluss von Kleinhirnfehlbildungen vorzunehmen, der wegen der Knochenüberlagerung sonografisch gelegentlich Schwierigkeiten bereiten kann. Die beiden häufigsten Fehlbildungen sind

- die Arnold-Chiari-Malformation sowie

- das Spektrum der Dandy-Walker-Malformation.

Arnold-Chiari-Malformation. Die Hauptkriterien der Arnold-Chiari-Malformation sind die kleine hintere Schädelgrube, die in den Spinalkanal reichenden Kleinhirntonsillen sowie die begleitende Myelomeningozele, die auf jeder Höhe des Spinalkanals auftreten kann. In den meisten Fällen ist ein Hydrozephalus nachweisbar (Abb. 18 und 19).

Dandy-Walker-Malformation (DWMF). Diese Störung ist dagegen durch eine vergrößerte hintere Schädelgrube mit Flüssigkeitssignal charakterisiert. Im typischen Fall handelt es sich um eine Hypoplasie des Vermis mit zystischer Dilatation des 4. Ventrikels und angehobenem Tentorium. Häufig liegt auch ein Hydrozephalus vor (Abb. 20) [16].

\section{Tipp für die Praxis}

Bei der Dandy-Walker-Malformation sollte nach assoziierten Fehlbildungen wie Balkendysgenesien oder kortikalen Dysplasien gefahndet werden.

Nicht in jedem Fall ist es möglich, die Zystenwand des 4. Ventrikels innerhalb des Liquors nachzuweisen. In diesen Fällen kann es sich um einen DWMF-ähnlichen Befund handeln, der unter dem etwas verschwommenen Begriff der Dandy-Walker-Variante subsummiert wird.

Die Megacisterna magna wird als Minimalvariante des Dandy-Walker-Komplexes angesehen. Hierbei ist der 4. Ventrikel nicht erweitert, der Vermis ist nicht hypoplastisch, ein Hydrozephalus fehlt.

Das MRT muss die klinisch relevante Unter-

scheidung einer DWMF zu einer Arachnoidalzyste treffen. 


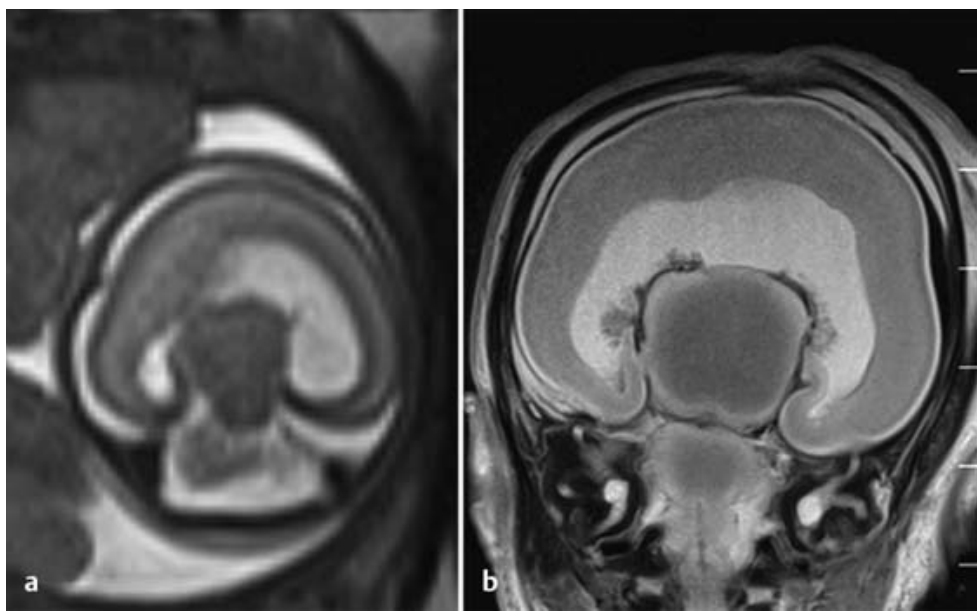

Abb. 14 a und b Alobare Holoprosenzephalie als Maximalform einer Fusionsstörung: vollständige Verschmelzung der beiden Hemisphären bei fehlender Falx, Monoventrikel ohne Septum; a: pränatales MRT, b: Bestätigung der Diagnose im postmortalen MRT.

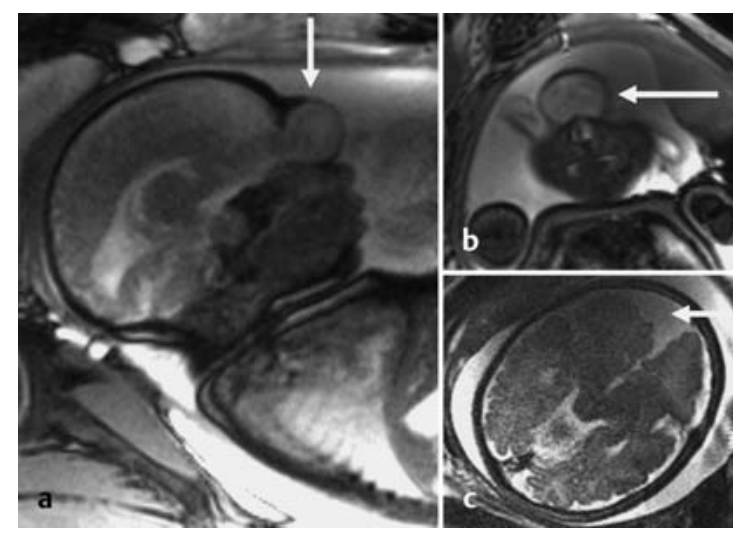

Abb. 15a bis c Frontale Meningoenzephalozele mit knöchernem Defekt im Bereich der Stirn (a). Oberhalb der Nasenwurzel wölbt sich der Zelensack nach außen vor (b). Der rechte Frontallappen ist in den frontalen Zelensack verlagert und daher nicht mehr an typischer Stelle nachweisbar (c).

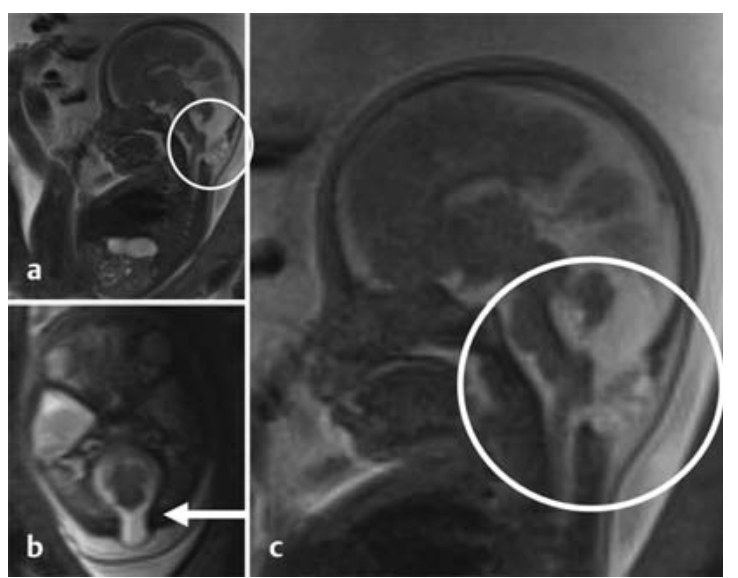

Abb. 16a bis c Okzipitale Meningozele (Kreis). Die Kalotte weist in dieser Lokalisation einen Defekt auf (Pfeil).

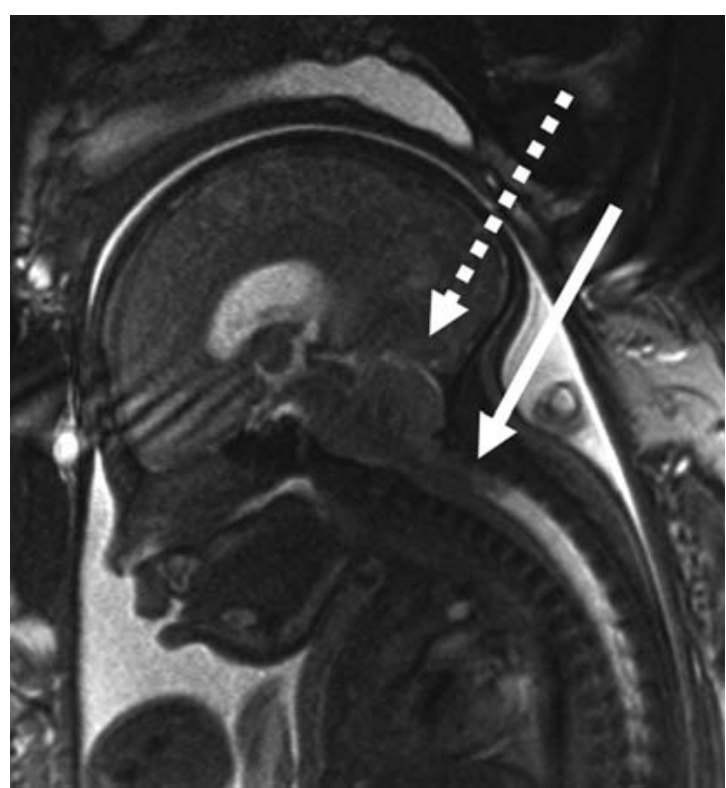

Abb. 18 Arnold-Chiari-Malformation mit Tiefstand der Kleinhirntonsillen im zervikalen Spinalkanal bei einem Fetus der 36. SSW (weißer Pfeil). Die hintere Schädelgrube ist deutlich verkleinert, das Tentorium setzt zu tief an (gestrichelter Pfeil).

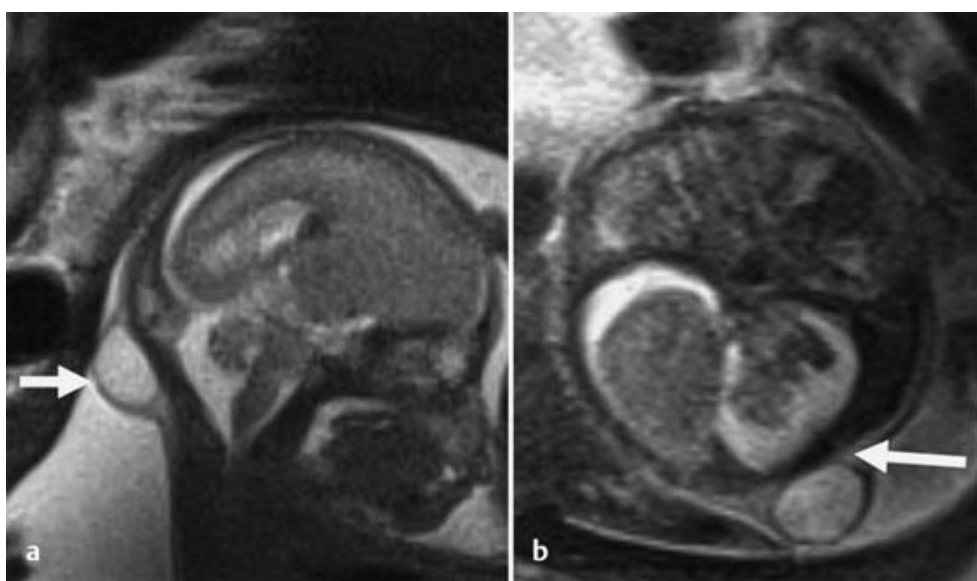

Abb. 17a und b Dysontogenetische Zyste okzipital in der 20. SSW. Im Gegensatz zur Differenzialdiagnose der Meningozele ist hier die darunterliegende Kalotte intakt.

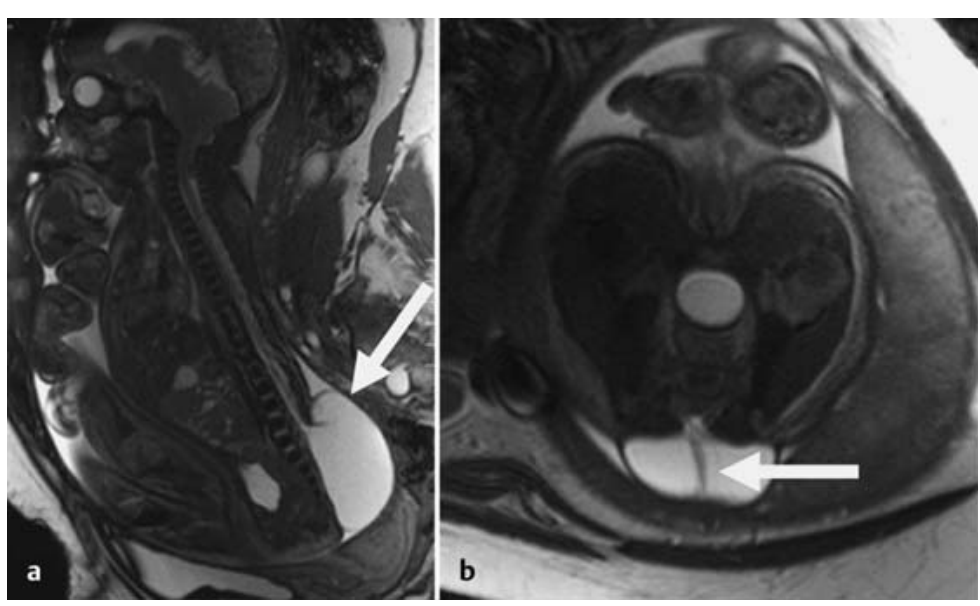

Abb. 19a und b Gleicher Patient wie Abb. 18. Darstellung der großen lumbosakralen Myelomeningozele mit Insertion der Rückenmarksfasern in der Plakode (Pfeil). 


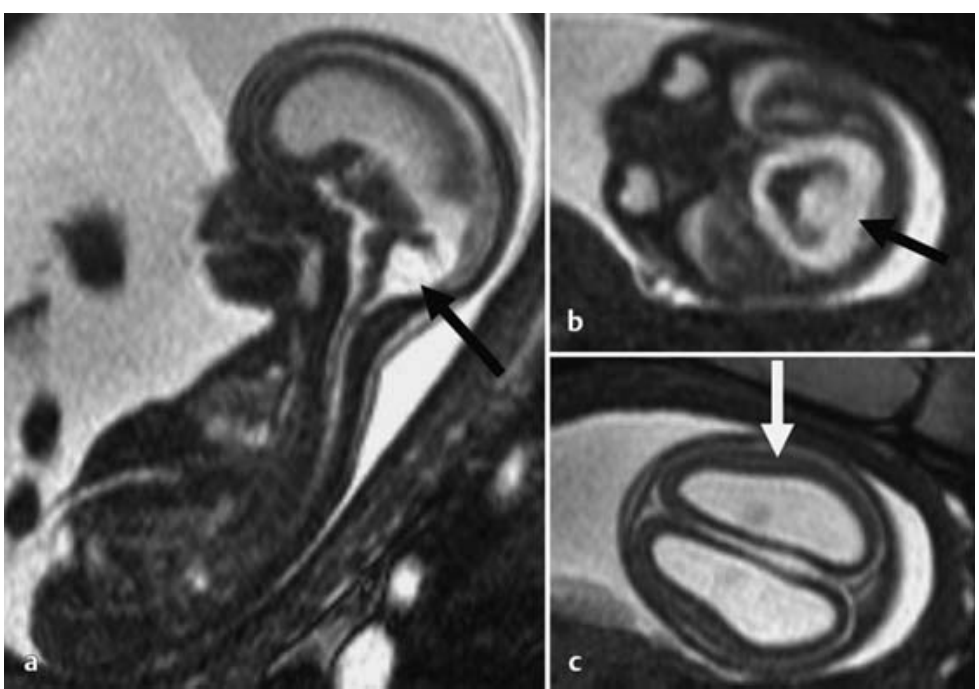

Abb. 20a bis c Dandy-Walker-Malformation mit Hydrozephalus in der 22. SSW: hochgradige Vermishypoplasie, Tentoriumhochstand, zystische Dilatation des 4. Ventrikels nach okzipital (schwarzer Pfeil), Ventrikeldilatation.
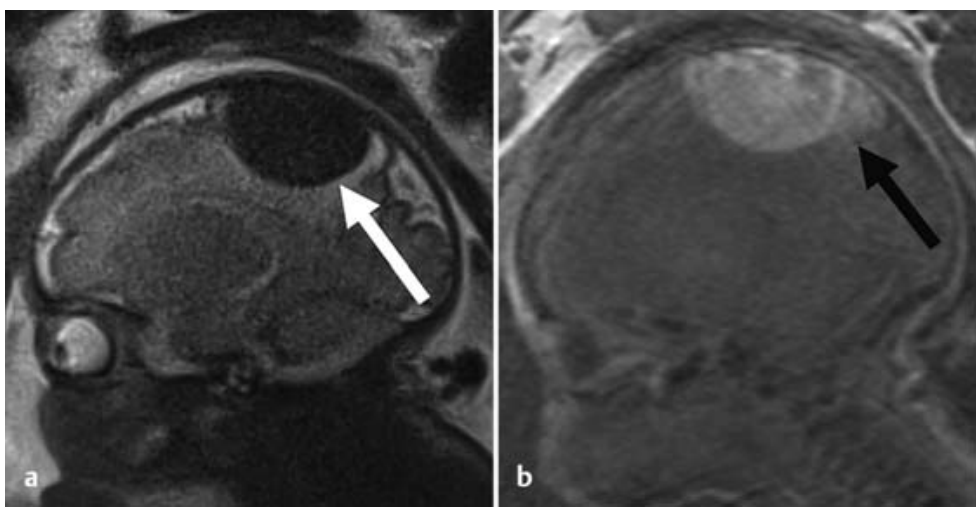

Abb. 21 a und b Epidurales Hämatom, 32. SSW. Das frische Blut stellt sich in der T2-Wichtung dunkel (a), in der T1-Wichtung hell (b) dar.
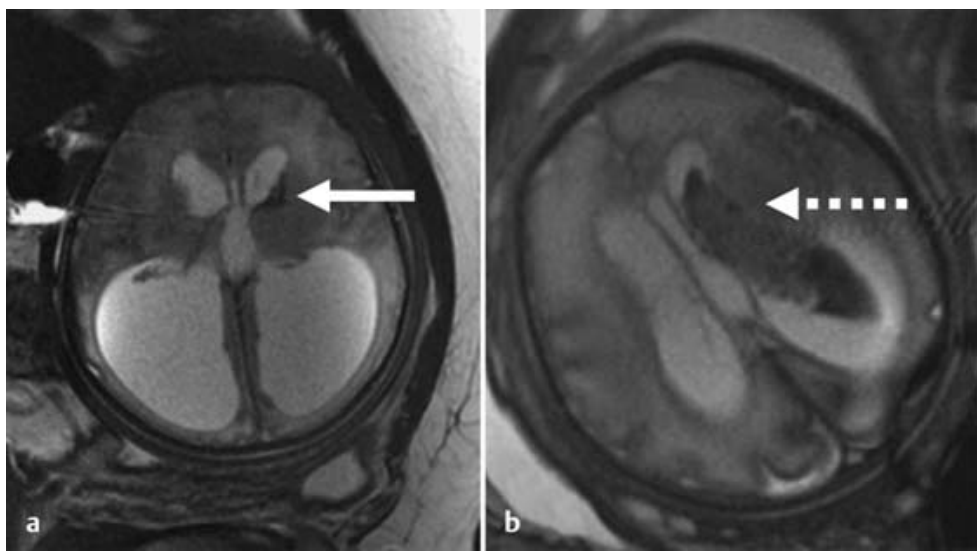

Abb. 22a und b Intrakranielle Blutung in der 35. SSW. a Die subependymale Blutung ist erkennbar an dem dunklen Signal in der nukleothalamischen Furche (Pfeil). Deutliche hinterhornbetonte Erweiterung der Seitenventrikel und erweiterter 3. Ventrikel.

b intraventrikuläre Blutung Grad III mit einem großen Koagulum im linken Seitenventrikel (gestrichelter Pfeil), konsekutiver posthämorrhagischer Hydrozephalus.
Im Unterschied zur DWMF komprimiert die Arachnoidalzyste das Kleinhirn von hinten, der 4. Ventrikel ist schmal und z.T. nach vorn verlagert [17].

Auch der fehlende Nachweis des Kleinhirnwurms ist von hoher klinischer Relevanz für das Kind und die Eltern. Im Falle einer Verschmelzung der Hemisphären handelt es sich um eine Rhombenzephalosynapsis. Im Falle einer Aplasie oder einer höchstgradigen Hypoplasie kann ein Joubert-Syndrom vorliegen. Typisch sind folgende Hinweise:

- das „Molar-Tooth“-Zeichen - das Mesenzephalon stellt sich auf den Transversalschnitten wie ein Backenzahn dar,

- die verdickten abgerundeten Pedunculi cerebellares. Die Hemisphären liegen dann aneinander, sind aber getrennt.

\section{Tipp für die Praxis}

Die Beurteilung der hinteren Schädelgrube ist bei zunehmender Kalottenkalzifizierung und ausgeprägtem Beckentiefstand des Kopfes mit dem MRT sicher möglich. Sie ist daher eine sinnvolle bildgebende Option bei sonografisch vermuteter Kleinhirnpathologie in der späten Schwangerschaft.

\section{Pränatal erworbene Hirnerkrankungen im MRT}

\section{Blutungen}

Pränatal auftretende Blutungen sind im MRT wegen der klaren Sequenzabhängigkeit der Blutabbauprodukte deutlich spezifischer zu identifizieren als im Ultraschall. Frische Blutungen sind dunkel in der T2-Wichtung und hell in T1-Wichtung (Abb. 21).

In Analogie zu der Klassifikation der Blutungen bei Frühgeborenen werden 3 Blutungstypen unterschieden:

- subependymale Blutung

- Ventrikelblutung

- Parenchymeinblutung

Die am häufigsten auftretende Blutung ist die subependymale Blutung (ICB Grad I, Abb. 22a), meistens in der nukleothalamischen Furche nachweisbar. Wenn die subependymalen Blutungen sekundär in das Ventrikelsystem einbrechen, resultiert die klassische Ventrikelblutung, die dann oft mit einem Hydrozephalus verbunden ist (Abb. 22 b). Die Parenchymeinblutung 


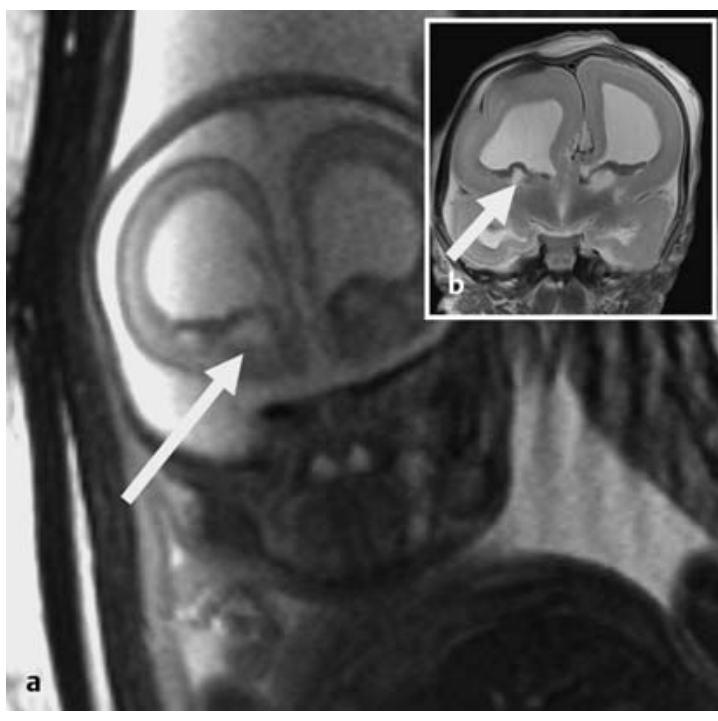

Abb. 23a und b Bilaterale Thalamusnekrose nach CMV-Infektion (a, Pfeil). Hydrozephalus internus, kleines Bild (b): Das postmortale MRT nach der Schwangerschaftsbeendigung bestätigt die Diagnose der lokalen Thalamusnekrose.

entspricht dem Typ IV der neonatalen ICB-Klassifikation.

\section{Tipp für die Praxis}

Blutungen ins Hirnparenchym und in die äußeren Liquorräume haben ein charakteristisches Signalverhalten und sind daher im MRT eindeutig von anderen pathologischen Läsionen zu differenzieren.

\section{Infektionen und Infarkte}

Das fetale Hirnparenchym reagiert auf Noxen, Infektionen und ischämische Alterationen recht uniform mit der gleichen Reaktion: Es entwickeln sich Kolliquationsnekrosen (Abb. 23). Am häufigsten finden sich kleine diffuse unspezifische Nekrosen. Nach Gewebeuntergang kann es hier auch zu Verkalkungen kommen, die öfter bei Infektionen (CMV, Toxoplasmose etc.) beschrieben werden, die aber nicht spezifisch hierfür sind.

Im Fall von Infarkten können die Nekrosen gelegentlich auch definierten Gefäßterritorien zugeordnet werden.

Die schwerste Form der generalisierten Hirnnekrotisierung ist die Hydranenzephalie (Abb. 24).

Eine sichere ätiologische Zuordnung solcher Läsionen ist auch mit MRT oft nicht möglich oder wenn, dann im
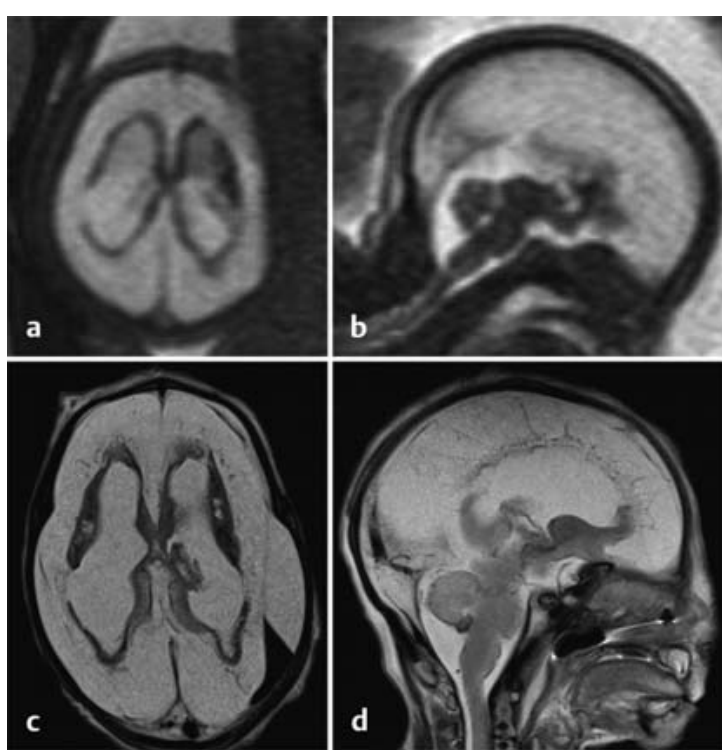

Abb. 24a bis d Fast vollständiger Untergang des Großhirngewebes in der 28. SSW a und $\mathbf{b}$ pränatales MRT; $\mathbf{c}$ und $\mathbf{d}$ virtuelle Autopsie nach Schwangerschaftsbeendigung; komplette nekrotische Umwandlung des Großhirns, während Hirnstamm und Kleinhirn strukturell unauffällig erscheinen.

Kontext und in Kenntnis der Rahmenbedingungen (Infektionsparameter, Plazentapathologie etc.). Im Einzelfall können Diffusionswichtungen helfen, die postischämische Genese zu belegen, jedoch gelingt das wegen der Länge der Diffusionsmessung nur bei ausgewählten ruhigen Feten. Dieser Befund stellt zudem nur die morphologische Endreaktion, aber nicht die Ursache der Ischämie dar [18].

\section{Hirntumoren}

Es kommen fast ausschließlich 2 Entitäten vor;

- Teratom

- supratentorieller primitiver neuroektodermaler Hirntumor (PNET)

Im Ultraschall ist gelegentlich die Differenzierung zwischen Hirntumor und Blutung nicht eindeutig. Im MRT gelingt dieses dagegen aufgrund des Weichteilkontrasts leicht. Eine MRT-Kontrastmitteluntersuchung mit Gadolinium ist zwar prinzipiell in solchen Fällen als Einzelfallentscheidung möglich, jedoch nur, wenn die Diagnose nicht schon vor KM-Gabe eindeutig erscheint (Off-Label-Use des Kontrastmittels). Es ist dabei weiterhin zu beachten, dass MR-Kontrastmittel nur gering plazentagängig sind und bei Hirntumoren oft keine oder nur eine geringe Kontrastmittelaufnahme nachweisbar ist (Abb. 25).

Die ausgeprägte Parenchymdestruktion und die sich im MRT sehr unterschiedlich darstellenden Gewebeanteile sind typisch für fetale Hirntumoren. 
Abb. 25 a bis $\mathbf{d}$ Pränataler Hirntumor (histologisch PNET). Links (a, c) pränatales MRT in der 32. SSW, rechts postnatales MRT im Alter von 2 Wochen. $\mathbf{a}$ und $\mathbf{b}$ T2-Wichtungen: sehr heterogener Tumor mit zystischen und soliden Anteilen, perifokalem Ödem und Mittellinienverlagerung. Abb. $\mathbf{c}$ und $\mathbf{d}$ T1-Wichtung nach Kontrastmittelgabe: inhomogenes Enhancement der soliden Tumoranteile.

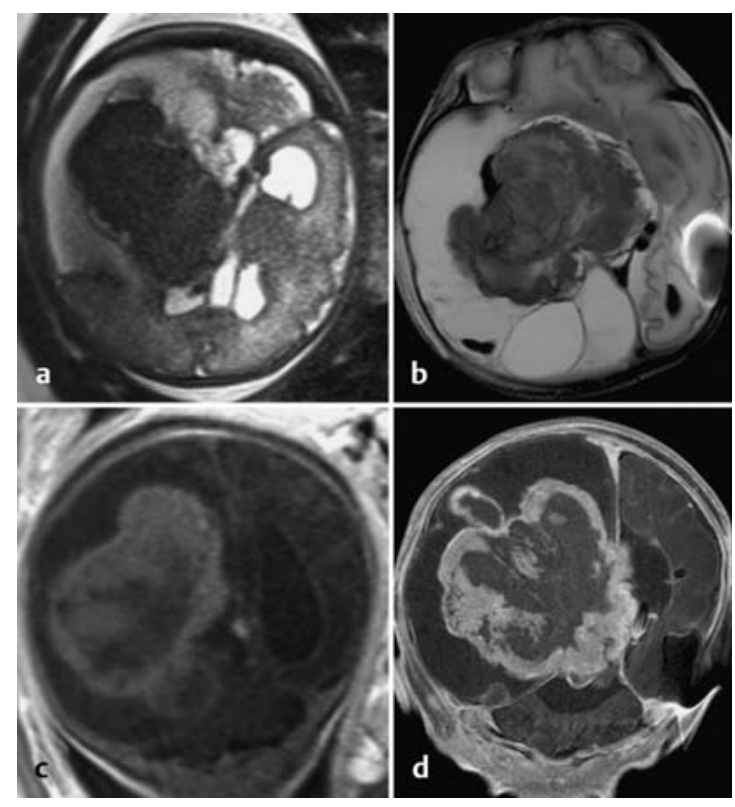

\section{Thorax}

\section{Lungenfehlbildung im fetalen MRT}

Das normale Lungengewebe stellt sich im MRT homogen und von leicht erhöhter Signalintensität dar. Die flüssigkeitsgefüllten Atemwege sind etwas besser im MRT darstellbar als im Ultraschall, dagegen ist das Herz wegen der Bewegungsunschärfe bisher nicht in einer Routineuntersuchung diagnostisch zu beurteilen. Die Signalintensität der Lunge nimmt in T2-Wichtung mit dem Reifegrad der Lunge zu und ist damit ein indirekter Lungenreifeparameter (Abb. 26).

Typische kongenitale Entwicklungsanomalien der Lungen sind:

- seltene Atresien (Larynx, Trachea, Bronchien)

- kongenitale Lungen-Atemwegs-Malformation CPAM (bisher als CCAM bezeichnet)

- bronchopulmonale Sequestration

Die kongenitale lobäre Flüssigkeitsüberladung (CLFO) ist eine spontan regrediente Lungenauffälligkeit und daher gegen die vorgenannten Entitäten abzugrenzen. Andere Fehlbildungen (Single thoracic Cysts, AV-Malformationen) sind sehr selten ( $<10 \%$ der Kinder) [19].

Die häufigsten Fehlbildungen scheinen eine gemeinsame entwicklungsgeschichtliche Grundlage zu haben, nämlich eine Atemwegsobstruktion zu unterschiedlichen Zeitpunkten der Embryonalentwicklung. Dies führt zu differierenden Erscheinungsbildern, aber auch

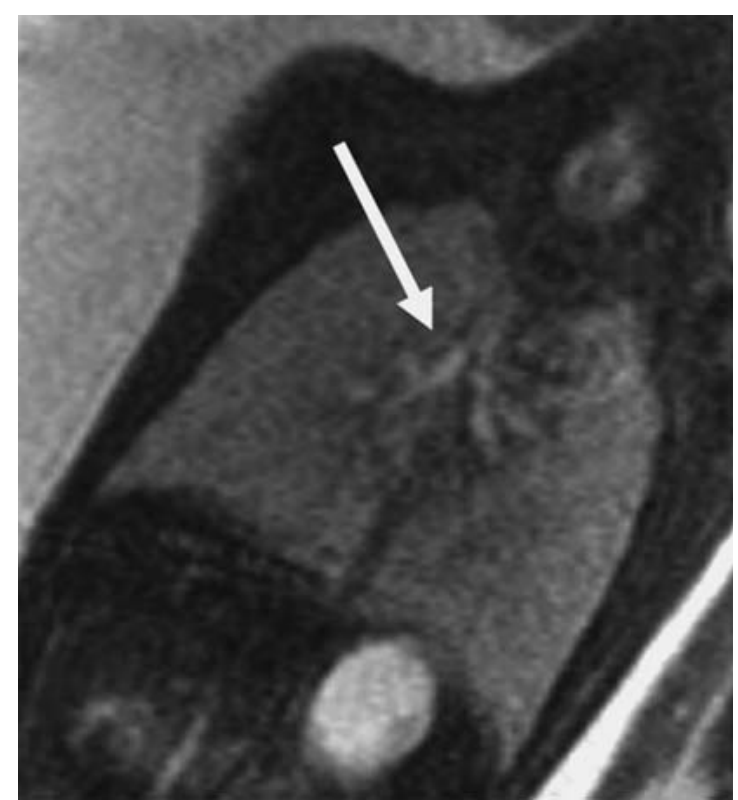

Abb. 26 Normale Lungendarstellung im MRT in der 22. SSW. Die Signalintensität der Lunge korreliert in T2-Wichtung mit dem Reifezustand der Lunge. Die flüssigkeitsgefüllten Atemwege (Pfeil) grenzen sich durch ihr helles Signal ab.

zu Mischformen. Wegen dieser Überschneidungsbereiche scheint eine pränatale MRT-Differenzierung von CPAM und Sequestration bildgebend nicht mehr so sicher möglich, wie man dies noch vor wenigen Jahren annahm. Dennoch sind tendenziell die im Folgenden beschriebenen Fehlbildungsentitäten zu unterscheiden.

\section{Zystisch adenomatoide Malformation der Lunge}

Zystisch adenomatoide Malformationen der Lunge (Congenital pulmonary Airway Malformation = CPAM, früher CCAM genannt) sind am häufigsten. Bis zu 50\% der Lungenfehlbildungen entfallen auf diese hamartösen Malformationen. Sie erscheinen häufig primär lobuliert. In 95\% der Fälle treten sie unilateral auf und zudem in $85 \%$ in nur einem Lungenlappen, meist im Unterlappen (Abb. 27).

Abweichend von der postnatalen Röntgen-Klassifikation wird im pränatalen MRT lediglich eine Unterteilung in mikrozystische $(<5 \mathrm{~mm})$ und makrozystische CPAM ( $>5 \mathrm{~mm}$ ) vorgenommen (Abb. 27). Das Risiko eines Hydrops (10-75\%), eines Polyhydramnions oder eines Mediastinalshifts ist wegen des größeren Volumeneffekts bei makrozystischer CPAM naturgemäß größer. 


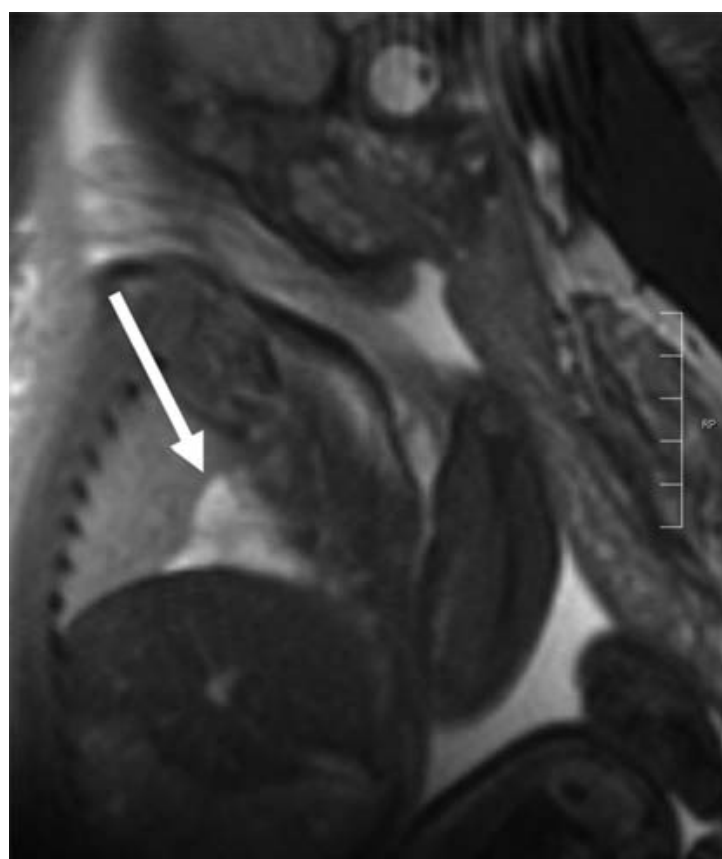

Abb. 27 Congenital pulmonary Airway Malformation (CPAM). Fetus der 28. SSW - die großzystischen Veränderungen des Mittellappens sind gegenüber der Lunge an ihrem helleren Signal zu erkennen.

CPAM können in $50 \%$ spontan regredient sein, die Regression setzt nach der 28. SSW ein.

\section{- Lungensequester}

Lungensequester sind die zweithäufigsten Lungenfehlbildungen. Sie sind scharf begrenzte oder lobulierte, homogene, hyperintense Raumforderungen. Sie sind definiert durch einen fehlenden Anschluss an das Bronchialsystem und eine versorgende Arterie aus der thorakalen Aorta oder der abdominellen Aorta. Dieses Gefäß zeigt einen Signalverlust (Signal Void) und bildet sich daher im MRT strichförmig signalarm ab, wenn es in der Schnittebene liegt. Dadurch gelingt eine Abgrenzung zur CPAM/CCAM.

Meist liegt eine Kombination von CPAM und Sequester im Sinne einer Hybridfehlbildung vor, was die eindeutige Zuordnung erschwert.

Falls spontan eine Regredienz des Sequesters eintritt, zeigt sich das durch eine zunehmende Randunschärfe und abnehmende Signalintensität im 3. Trimenon. Lungensequester können atypischerweise auch im Bereich des oberen Abdomens vorkommen.

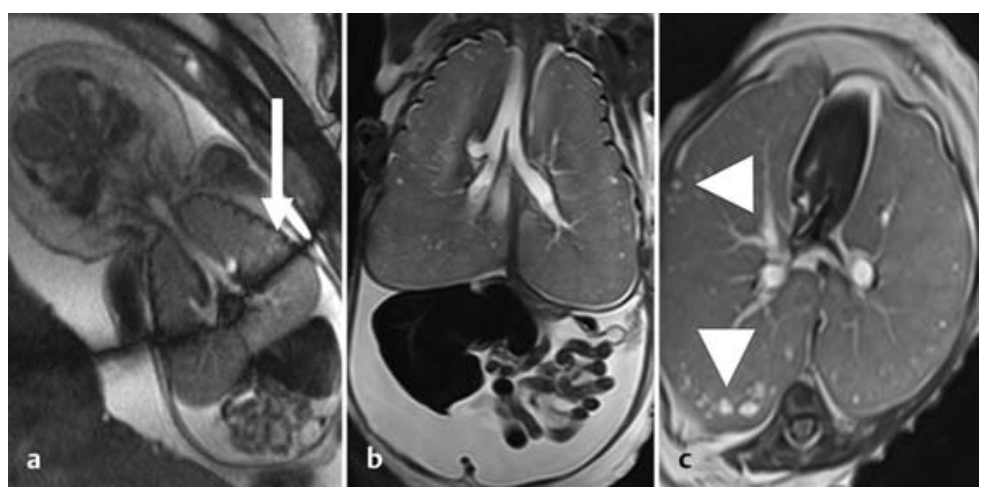

Abb. 28 a bis c Larynxatresie bei einem Fetus der 19. SSW. a Das pränatale MRT zeigt das erweiterte Tracheobronchialsystem, die erheblich vergrößerten, nach kaudal konvexen Lungen, Aszites sowie einen generalisierten Hydrops. Angedeutet sind auch kleine periphere Lungenzysten erkennbar (Pfeil). b und c zeigen ein postmortales MRT im Rahmen der virtuellen Autopsie nach Schwangerschaftsbeendigung. Erkennbar sind die peripheren Lungenzysten (Pfeilspitze) als Ausdruck der Lungenparenchymschädigung sowie das extrem schmale, durch die vergrößerten Lungen komprimierte Herz.

\section{Congenital Lobar Fluid Overload}

Der Congenital Lobar Fluid Overload (CLFO) zeigt einen einseitigen flüssigkeitsüberladenen Lungenlappen ohne strukturelle Anomalie. Die Lungengefäße sind gestreckt. Der Befund normalisiert sich meist im 3. Trimenon.

\section{Atresie der Atemwege}

Eine Atresie der Atemwege (Congenital high Airway Obstruction, CHAOS) führt zu einer Überladung beider Lungen mit der sezernierten Flüssigkeit des Lungenparenchyms, die nicht über die Trachea abfließen kann. Die Folge sind bilateral prall überfüllte Lungen, die das Zwerchfell konvex nach abdominal vorwölben und zwischen den Rippen hindurch prolabieren. Zudem können kleine periphere Zysten auf die Lungenparenchymschädigung hinweisen (Abb. 28).

Wenn der Verschluss komplett ist, z. B. durch eine laryngotracheale Atresie, so ist die Prognose infaust. Wenn jedoch eine druckentlastende Fistel zum Ösophagus besteht, ist die Symptomatik wenig ausgeprägt. Ein eindeutiger Ausschluss einer Fistel gelingt aber auch mit der MRT nicht. In der Regel ist die Höhenlokalisation des Verschlusses im MRT möglich [20]. 
Kasuistik zum fallorientierten Lernen

\section{Anamnese}

Eine 21-jährige Erstgravida wurde zur Einholung einer Zweitmeinung im Ultraschall (DEGUM II) vorgestellt; im Anschluss daran erfolgte die Überweisung zum fetalen MRT. Vorausgegangen war eine Amniozentese bei hyperechogenem Darm und Ventrikulomegalie. Es ergab sich hierbei kein pathologischer Befund. Im Ultraschall in der 22. + 0 SSW lag der Kopfumfang unter der 5. Perzentile, und unterhalb des Interhemisphärenspalts war eine $11 \mathrm{~mm}$ große Zyste nachweisbar. Das Corpus callosum war bei dorsoposteriorer Kindslage nicht sicher darzustellen. Die Fragestellung an das MRT war der Ausschluss bzw. der Nachweis einer möglichen Balkenagenesie und ggf. der Nachweis weiterer Begleitfehlbildungen.

\section{Befund}

Das MRT wurde in der 23. + 2 SSW durchgeführt. Dabei zeigte sich eine geringe Hinterhornbetonung mit $9 \mathrm{~mm}$ bei jedoch unauffälligen Seitenventrikelvorderhörnern. Der Balken war im Bereich des vorderen Balkenknies und des Corpus sicher nachweisbar, das Splenium stellte sich nicht eindeutig dar. Es fand sich weiterhin die sonografisch vorbeschriebene, $1 \mathrm{~cm}$ große interhemisphärische Zyste okzipital ohne Kommunikation mit dem Ventrikelsystem (Typ IIa). Neben diesen, den Ultraschall bestätigenden Befunden stellten sich mehrere Spaltbildungen im Sinne einer Open-Lip-Schizenzephalie dar: rechts okzipital und rechts parietal sowie links temporal (s. Abb. K1).

In der 24. SSW sollten sowohl der Sulcus parietooccipitalis als auch der Sulcus calcarinus schon abgrenzbar sein. Da das beim Fetus noch nicht der Fall war, musste zusätzlich der dringende Verdacht auf eine Gyrierungsstörung geäußert werden. Prinzipiell besteht ein enger Zusammenhang zwischen Gyrierung und Migration. In der 24. SSW ist die Migration jedoch auch physiologischerweise noch nicht fortgeschritten, der frühe zonale Aufbau des Hirnmantels war noch deutlich erkennbar, sodass ein Migrationsarrest beim Fetus noch nicht verlässlich diagnostizierbar war.

\section{Diagnose}

Mehrfache Open-Lip-Schizenzephalie, V. a. Gyrierungsstörung, interhemisphärische Zyste Typ Ila bei Balkenhypogenesie.

\section{Verlauf}

Durch die im MRT zusätzlich erhobenen Befunde einer doppelseitigen Schizenzephalie und den Verdacht auf eine Gyrierungsverzögerung verschlechterte sich die neuropädiatrische Prognose erheblich. In einem interdisziplinären Konsil wurden die Befunde gemeinsam von Neuropädiater, Gynäkologen und Kinderradiologen demonstriert. Es erfolgte eine Beratung bezüglich der Spannbreite der zu erwartenden klinischen Symptomatik. Zudem wurden die therapeutischen Möglichkeiten und die medizinischen und psychosozialen Hilfsangebote dargestellt. Die Frau sah sich trotz der ergebnisoffenen Beratung nicht in der Lage, die Schwangerschaft fortzusetzen und entschied sich für eine vorzeitige Schwangerschaftsbeendigung.
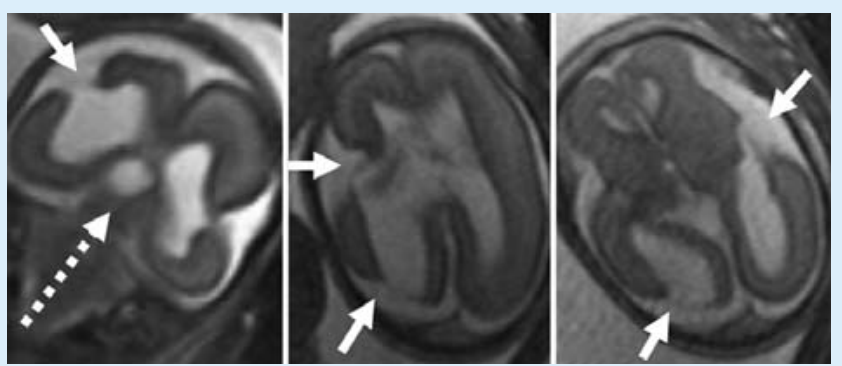

Abb. K1 23. + 2SSW: offene Spaltbildung links temporal und rechts parieta und okzipital (kleine Pfeile), interhemisphärische Zyste Typ II a (gepunkteter Pfeil).

\section{Kongenitaler Zwerchfelldefekt (Zwerchfellhernie)}

Die Prognose der Zwerchfellhernie mit ihrer noch immer hohen Mortalität hängt fast ausschließlich von der pulmonalen Hypoplasie und der damit korrelierenden strukturellen Lungenveränderung ab. Die Prognoseparameter sind daher heute an das Lungenvolumen der kontralateralen und der ipsilateralen Restlunge gebunden [21]. Das Volumen der ipsilateralen Lunge ist sonografisch nur in der Hälfte der Fälle bestimmbar [22]. Mit der MRT wird sowohl das Volumen der unbeeinträchtigten Lunge als auch das der Restlunge auf der ipsilateralen Seite als absolutes Volumen gemessen und ins Verhältnis gesetzt zum erwarteten Lungenvolumen der entsprechenden Altersgruppe. Anhand dieses Wertes ist eine Einteilung in eine Low-Risk- und eine HighRisk-Gruppe möglich; entsprechend sind die Prognoseaussichten und die Aggressivität der notwendigen Therapie zu wählen [23].

\section{Die MRT eignet sich zur Vermessung der Volumina} beider Lungenflügel.

Ein weiterer Prognosefaktor ist die Lage der Leber, deren Position sicher pränatal bestimmt werden muss. Mit dem MRT kann die Herniation des linken Leberlappens eindeutig visualisiert werden, da im Gegensatz 
zum US die Signalcharakteristik der Leber und der Lunge vollkommen different ist. Auch die Frage, ob Dickdarmanteile innerhalb der Herniation intrathorakal liegen, kann aufgrund des hellen Signals in T1 eindeutig beantwortet werden (Abb. 29).

\section{Tipp für die Praxis}

Bei einem kongenitalen Zwerchfelldefekt ist die MRT des fetalen Thorax eine notwendige Zusatzuntersuchung, um die anstehenden Therapieentscheidungen auf der Kenntnis einer bestmöglichen morphologischen Basis zu treffen.

\section{Kongenitaler Hydrothorax}

Eine Unterscheidung zwischen kongenitalem Chylothorax und kongenitalem Hydrothorax ist durch den höheren Eiweißgehalt bei ersterem mit dem MRT möglich. Es handelt sich um Anomalien im Verlauf des Ductus thoracicus. Die pleurale Flüssigkeit ist dann in der T1-Wichtung signalreicher, aber nicht so hell wie frisches Blut.

Beim kongenitalen Hydrothorax liegt dagegen eine schwerwiegende Grunderkrankung vor, z. B. Herzfehler, Infektionen oder metabolische oder immunologische Störungen (= sekundärer Hydrothorax durch ein Dysäquilibrium an den pleuralen Membranen). Trotz einer systemischen Erkrankung kann der Hydrothorax gelegentlich nur einseitig auftreten (Abb. 30). Die Prognose des sekundären Hydrothorax ist in Abhängigkeit von der zugrunde liegenden Erkrankung schlechter als beim kongenitalen Chylothorax. Beim ausgeprägten, lang dauernden Hydrothorax kann eine Lungenhypoplasie entstehen [24].

\section{Abdominelle und retro- peritoneale Fragestellungen}

MRT-Untersuchungen des fetalen Abdomens und Beckens sind in der täglichen Routine deutlich seltener als die des Kopfes und des Thorax. Sie machen nur 2-18\% aller fetalen MRT-Anforderungen aus [25]. Das MRT bietet aber gerade im Abdominalbereich einige methodenbedingte Vorteile in der Visualisierung der Organe: Durch das hohe Signal des Mekoniums in der T1-Wichtung ab der 20. SSW kann das Kolon sehr gut und selektiv signalreich in der MRT abgebildet werden (Abb. 31) [26]. Die Dünndarmschlingen und die pathologischen Befunde dieser Darmabschnitte sind dagegen
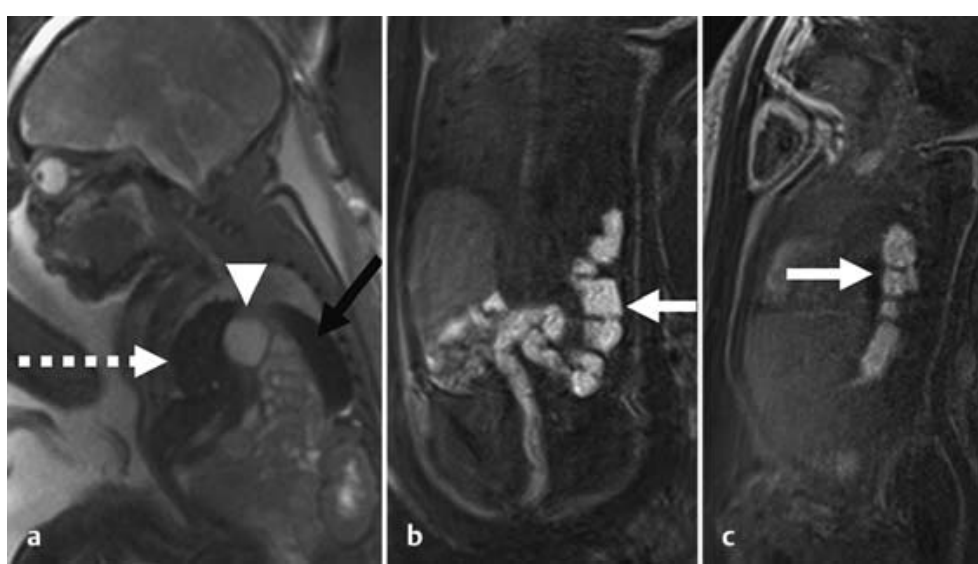

Abb. 29a bis c Zwerchfellhernie, 34. SSW. a Verlagerung von Leber (gestrichelter Pfeil), Magen (Pfeilspitze) und Milz (schwarzer Pfeil) in den linken Hemithorax. Unter dem Magen findet sich Dünndarm (T2-Wichtung). b In T1-Wichtung ist das Kolon durch das helle Mekonium gut zu erkennen (weißer Pfeil) und belegt, dass auch Teile des Kolons intrathorakal gelegen sind (c).

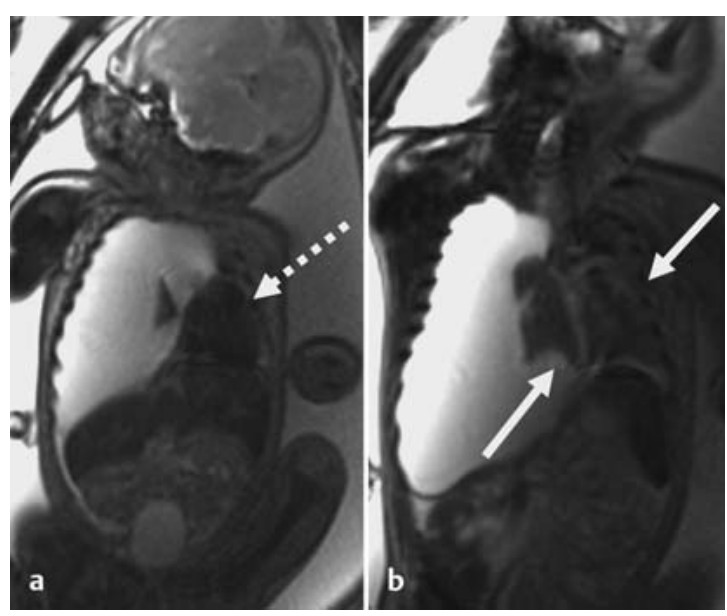

Abb. $\mathbf{3 0}$ a und $\mathbf{b}$ Ausgeprägter Pleuraerguss, rechts mehr als links, bei einem Fetus 33. SSW. a Deutlicher Zwerchfelltiefstand rechts und Verlagerung von Herz und Mediastinum zur Gegenseite (gestrichelter Pfeil). b Es resultiert eine erhebliche Lungenhypoplasie beidseits (Pfeile). Die Genese der Pleuraergüsse erschließt sich aus dem MRT nicht.

besser in der T2-Wichtung durch ihre Distension und Wasserfüllung nachweisbar. Allerdings können im Falle eines pathologischen Dünndarmbefundes auch hier in Einzelfällen hohe Signalintensitäten in der T1-Wichtung auftreten. Dieses wurde vor allem bei distaler Dünndarmatresie gefunden, aber auch bei der zystischen Fibrose. Der Weichteilkontrast des MRT ist in vielen Fällen eine wichtige Ergänzung bei folgenden Fragestellungen:

- Weichteiltumoren

- Infiltrationsbeurteilungen

- Organfehlbildungen

- Nierenparenchymveränderungen 


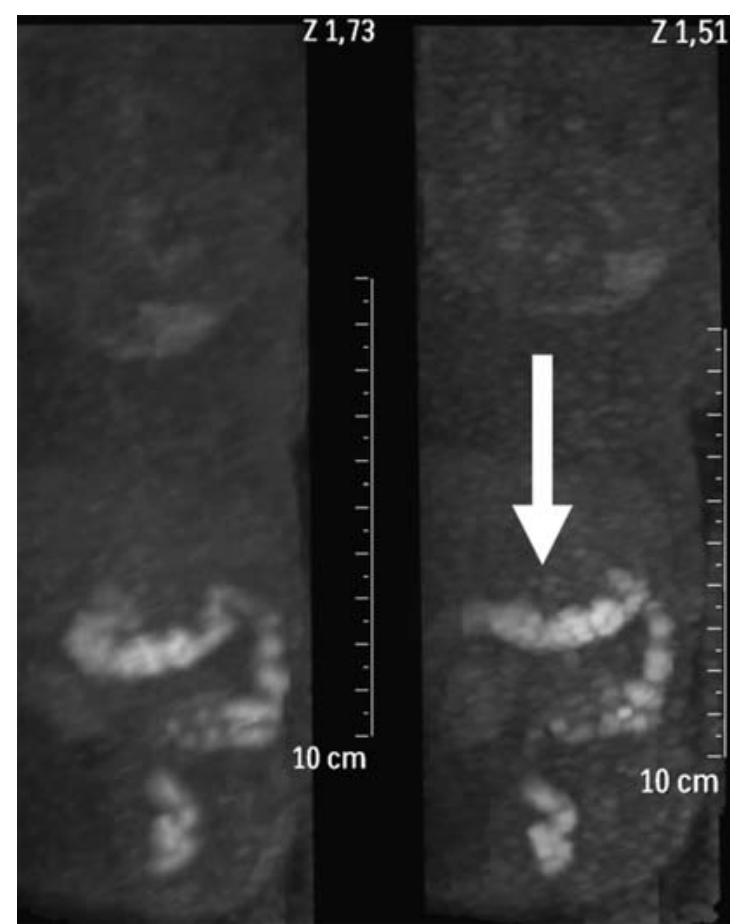

Abb. 31 „Kolonografie“ in der MPR-Darstellung: Das mekoniumgefüllte Kolon stellt sich in der T1-Wichtung hell dar, alle anderen Organe erscheinen dunkel im Hintergrund.

\section{Verlagerung von Darmschlingen}

Neben der kongenitalen Zwerchfellhernie ( $\mathrm{CDH}$, siehe Thoraxuntersuchungen) kommen Verlagerungen von Darmabschnitten bei der Gastroschisis und der Omphalozele vor (Abb. 32).
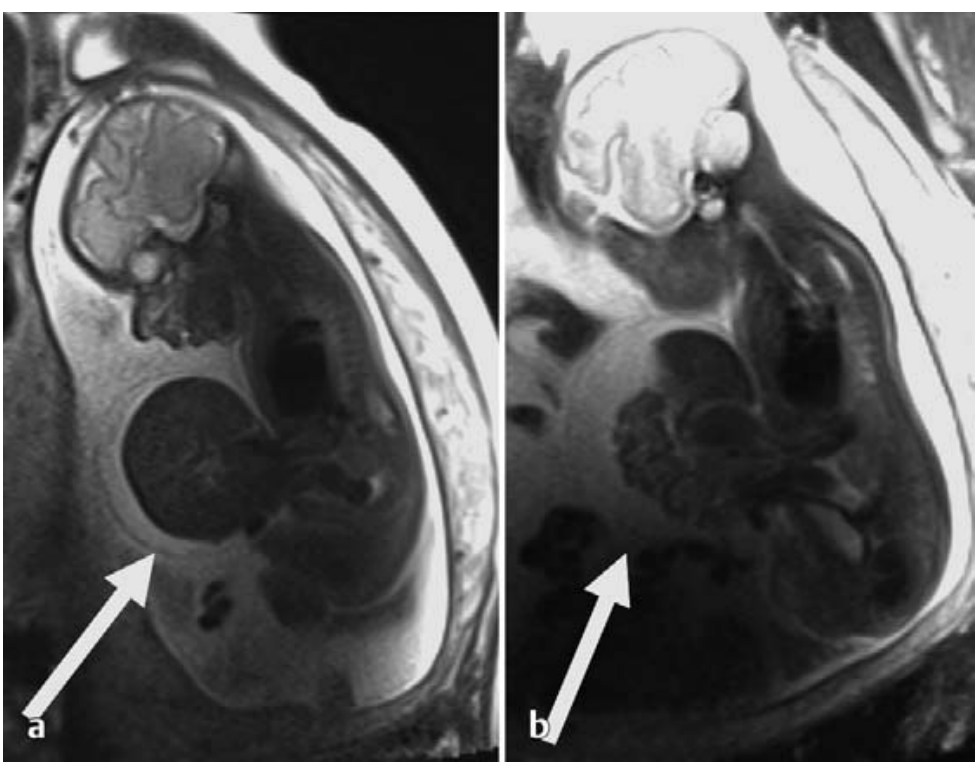

Abb. 32a und b Omphalozele: Das MRT zeigt die nach außen verlagerte Leber und den Dünndarm innerhalb des Zelensacks.

\section{Tipp für die Praxis}

Die Position der Darmanteile und der zu erwartende Bruchsackinhalt können wegen des hohen Weichteilkontrasts und des deutlich größeren „Field of View“ (FOV, im MRT bis zu $50 \mathrm{~cm}$ ) den kinderchirurgischen Partnern untersucherunabhängig und reproduzierbar demonstriert werden.

\section{Zystische abdominelle Raumforderungen}

Zystenbefunde im Abdomen stellen für alle bildgebenden Modalitäten eine Herausforderung dar. Der Ursprung der zystischen Läsion ist häufig auch im MRT nicht eindeutig zuzuordnen, dennoch wurde beschrieben, dass es zu therapierelevanten Mehrinformationen durch ein fetales MRT bei sonografisch unklaren Befunden kam [27]. Statistisch am häufigsten sind die Ovarialzysten (Abb. 33), die einbluten können und dann mit dem MRT sicher als blutgefüllt identifiziert werden können. Differenzialdiagnosen sind Mesenterialzysten, abdominelle Lymphangiome, Hydronephrose mit Megaureter und Hydrometrokolpos.

\section{Fehlbildungen des Magen-Darm-Traktes}

Hierbei handelt es sich um Atresien, Stenosen und Duplikaturen. Eine fehlende Magendarstellung lenkt zunächst stets den Verdacht auf eine Ösophagusatresie. Umgekehrt kann der Magen aber trotz einer Atresie

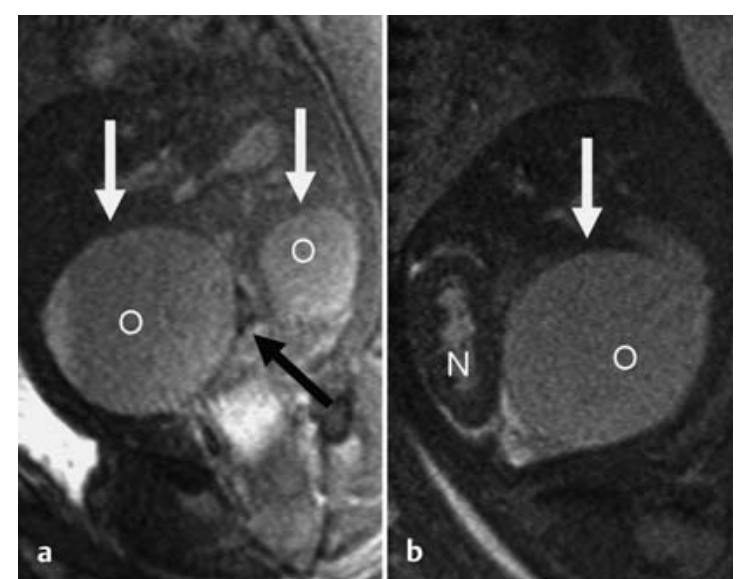

Abb. 33 a und b Große Ovarialzysten bds. (O, weiße Pfeile). a An der rechten Zyste ist ein signalarmer Anteil erkennbar (schwarzer Pfeil), der für eine Verkalkung spricht. b Im Sagittalschnitt ist die Lagebeziehung zur normalen Niere (N) gut erkennbar. Postnatal persistierte die rechte Zyste, links hatte sich die Zyste zurückgebildet. Die OP im Alter von 5 Monaten zeigte eine Schokoladenzyste rechts bei Z.n. Stieldrehung des Ovars. 


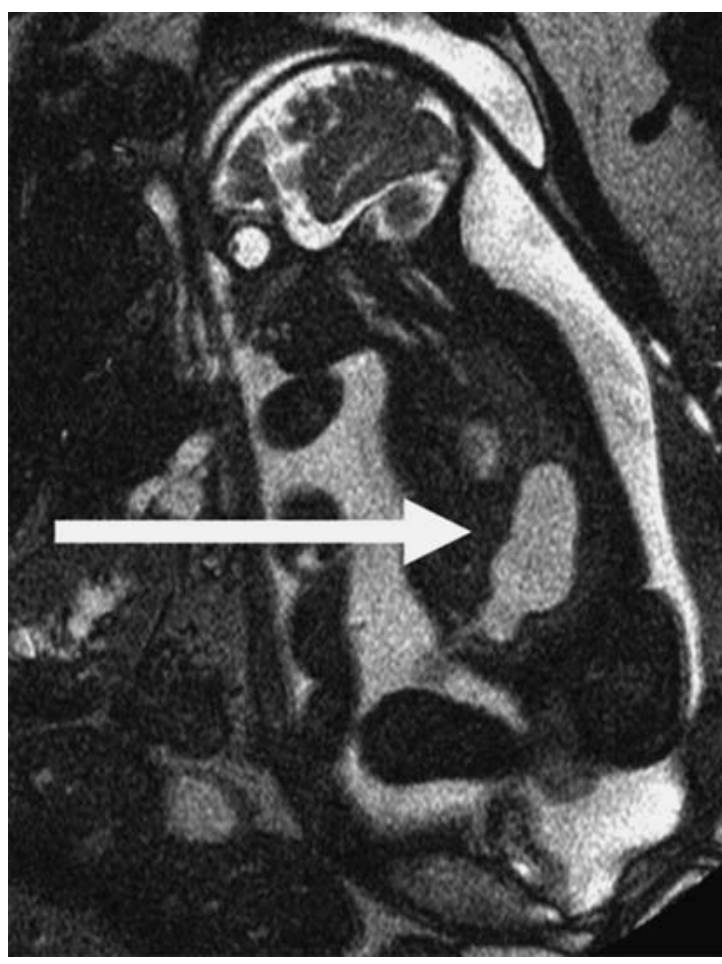

Abb. 34 Duodenalstenose: Im pränatalen MRT sind der große flüssigkeitsgefüllte Magen und der Bulbus duodeni erkennbar.

über die tracheoösophageale Fistel gefülltt sein. Ein sicherer Ausschluss einer Ösophagusatresie ist dann auch mit der MRT nicht möglich.

Die Duodenalatresie ist leicht an dem postnatalen bekannten „Double-Bubble-Phänomen“ zu erkennen, d. h. der vermehrten Flüssigkeit im dilatierten Magen und dem gleichzeitig ballonierten Bulbus duodeni (Abb. 34).

Ganz allgemein zeigt jede Atresie und jede hochgradige Stenose eine präatretische Dilatation.

Mithilfe von dynamischen T2-SSFP-Sequenzen kann ergänzend die Darmmotilität des Fetus gut visualisiert werden. Bei Stenosen liegt meistens eine Hyperperistaltik vor.

Die Darstellung der Kolonschlingen und der Pathologie des Kolons ist eine Domäne der MRT.

Durch das in T1-Wichtung sehr helle Signal des Mekoniums ist eine selektive Kolondarstellung möglich. Allerdings ist das Lumen des Kolons nicht immer homogen hell kontrastiert, was auf unterschiedliche Konzentrationen von Eiweiß und Mineralien des Mekoniums zurückgeführt wird. Durch eine Summation der Einzelschnittbilder kann zusätzlich ein räumlicher
Bildeindruck ähnlich wie bei einem Kolonkontrasteinlauf erreicht werden, was die Beurteilung sehr erleichtert (Abb. 31). Die hohe Form einer Analatresie ist daher sicher mit dem MRT zu erkennen, da das helle Mekoniumsignal im Rektum fehlt bzw. konvexbogig endet [28].

Bei den tiefen Formen der Analatresie und bei der (sehr häufigen!) ventralen Fistel zum Damm kann die Atresie gelegentlich dem MRT-Nachweis entgehen. Falls eine solche Fistel besteht, aber sich nicht zum Damm, sondern zur Blase oder zu einem Sinus urogenitalis öffnet, so ist dieses wiederum leicht an der Hyperintensität in $\mathrm{T} 1$ in der Blase und am gleichzeitig hellen Signal des Rektums in $\mathrm{T} 2$ zu diagnostizieren.

\section{Tipp für die Praxis}

Das Mekonium stellt sich in der T1-Wichtung isoliert hell (weiß) dar. Da es sich um ein kolontypisches spezifisches Signalverhalten handelt, ist es für die Erkennung von Kolonfehlbildungen oder Kolonverlagerungen ein sehr hilfreiches diagnostisches Kriterium.

\section{Tumoren im Abdominalbereich}

Teratome. Teratome, insbesondere die Ovarial- und Steißbeinteratome, sind die häufigsten abdominellen Tumoren bei Feten. Das MRT ermöglicht in diesen Fällen eine präzise Diagnose, wenn sich relevante Fettanteile innerhalb des Tumors befinden.

\section{Tipp für die Praxis}

Fett ist in allen Wichtungen (T1 und T2 und Mischsequenzen) signalreich, eine Eigenschaft, die kein anderes Körpergewebe aufweist. Da Fett anderseits nur in Teratomen zu finden ist, ist die Diagnose damit verlässlich mit dem MRT zu stellen.

Im Zweifelsfall kann eine fettunterdrückende Sequenz zur Gewebecharakterisierung herangezogen werden. Deshalb ist die ergänzende MRT-Bildgebung bei abdominellen Tumoren als Zweitmethode zur Artdiagnose eines Tumors wichtig geworden (Abb. 35). Zudem erlaubt die MRT wegen des hohen Weichteilkontrasts eine präzise Bestimmung der Infiltrationstiefe von Tumoren. Die Kenntnis der Organfiltration und der genauen Infiltrationstiefe ist v. a. bei pränatalen Eingriffen (z.B. ultraschallgestützte Radiofrequenzablation von Steißbeinteratomen) von Bedeutung für die Therapiewahl und -intensität. 


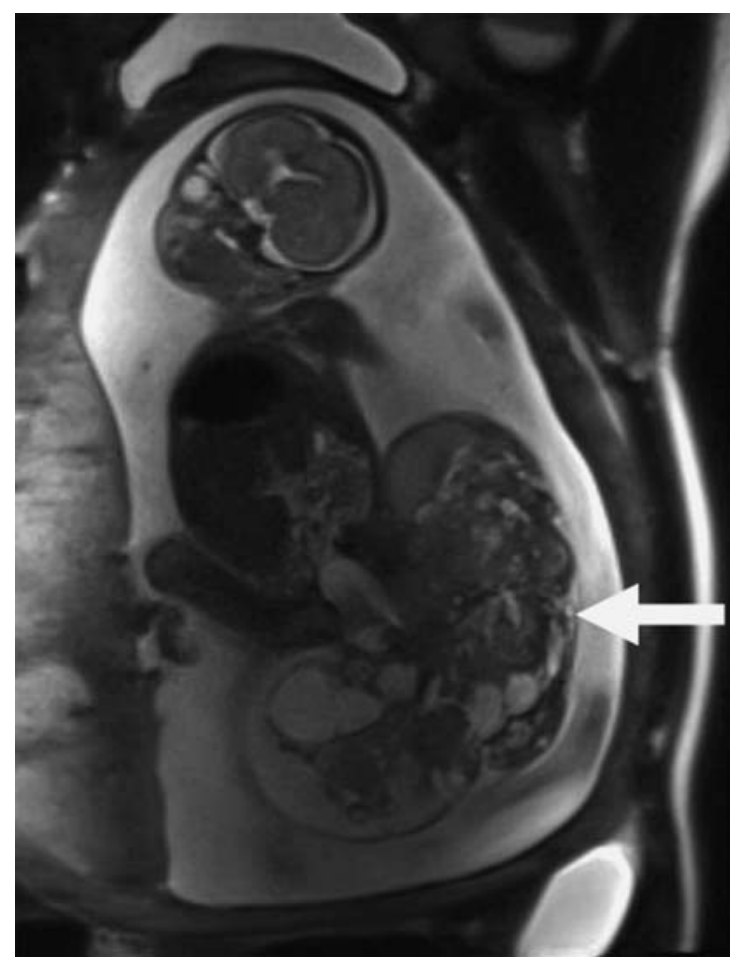

Abb. 35 Großes Steißbeinteratom bei einem Feten der 25. SSW.

Teratome lassen sich im MRT durch ihre Signalcharakteristik eindeutig diagnostizieren, aber nur, wenn sie einen Fettanteil haben.

Fetale Lebertumoren. Diese sind selten. 5\% aller fetalen Tumoren entfallen auf die Leber [29]. Im Wesentlichen handelt es sich dabei um vaskuläre Tumoren (Hämangioendotheliome und Hämangiome) und zystische mesenchymale Hamartome. In knapp 20\% der Fälle werden aber auch Hepatoblastome gefunden. Eine Differenzierung ist schwierig, jedoch sprechen rein zystische Läsionen am ehesten für ein mesenchymales Hamartom (Abb. 36). Diese Läsionen wachsen oft sehr schnell, sodass ggf. eine Schnittentbindung erwogen werden muss. Die Unterscheidung zwischen zystischem Charakter und gewebiger Grundstruktur einer Raumforderung lässt sich im MRT auch ohne Kontrastmittelgabe leichter als im US treffen.

Bei zystischen Lebertumoren handelt es sich fast ausschließlich um mesenchymale Hamartome.

\section{Nieren und urogenitale Erkrankungen}

Die Rolle des MRT bei urogenitalen Erkrankungen besteht im Nachweis bzw. der Differenzierung von Fehlbildungen bei einer zuvor sonografisch nachgewiese-

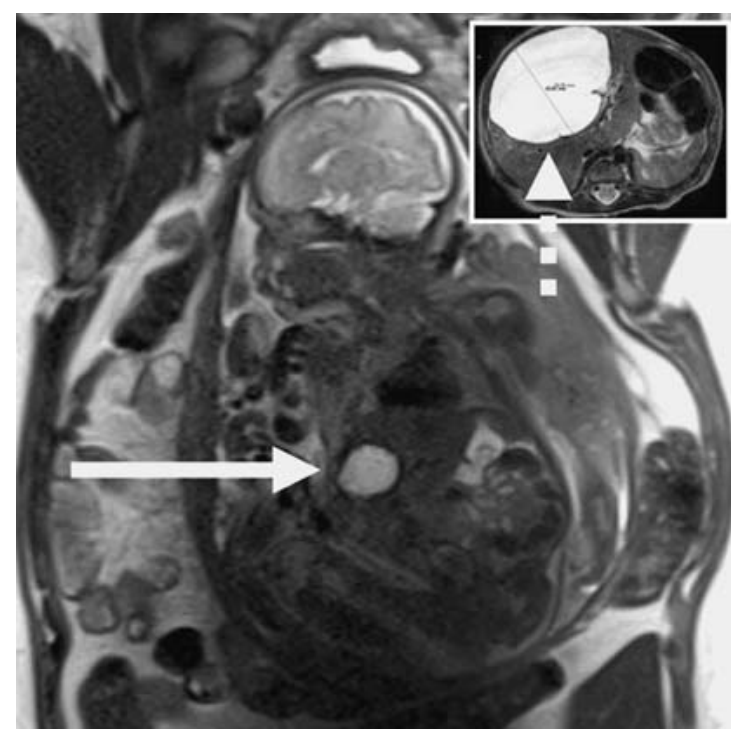

Abb. 36 Zystische Raumforderung in der Leber eines Feten der 33. SSW (Histologie: mesenchymales Hamartom). Das kleine Bild zeigt das MRT des Kindes im Alter von 4 Wochen. Die benigne Raumforderung hat deutlich an Größe zugenommen.

nen Anomalie. Durch gute Differenzierung der Flüssigkeiten in T2-Bildern ist insbesondere bei Stauungen und Zysten eine Zusatzinformation durch das MRT zu erwarten.

\section{Konnatale Hydronephrose}

Da sich die Ureteren sonografisch nicht ausreichend abbilden, ist das MRT in der ätiologischen Aufklärung der „kongenitalen Hydronephrose“ überlegen. In den meisten Fällen ist es mit dem MRT möglich, Urethralklappen, Ureterostiumstenosen oder vesikoureteralen Reflux als Ursache für eine pränatale Hydronephrose zu benennen und zu differenzieren [30].

\section{Nierenerkrankungen}

Die multizystisch dysplastische Niere (MCDK) ist ein relativ häufiger, meist einseitiger Befund. Sie ist häufig in Kombination mit einer kontralateralen distalen Ureterobstruktion und einem kontralateralen Megaureter zu finden. Sie tritt mit einer Inzidenz von 1:3500 auf und hat langfristig eine gute Prognose.

Die autosomal-rezessive polyzystische Nierenerkrankung (ARPCKD) tritt dagegen bilateral auf. In der perinatalen oder neonatalen Form hat sie eine schlechte Prognose. In der Hälfte der neonatalen Fälle liegt eine assoziierte Leberfibrose vor. 

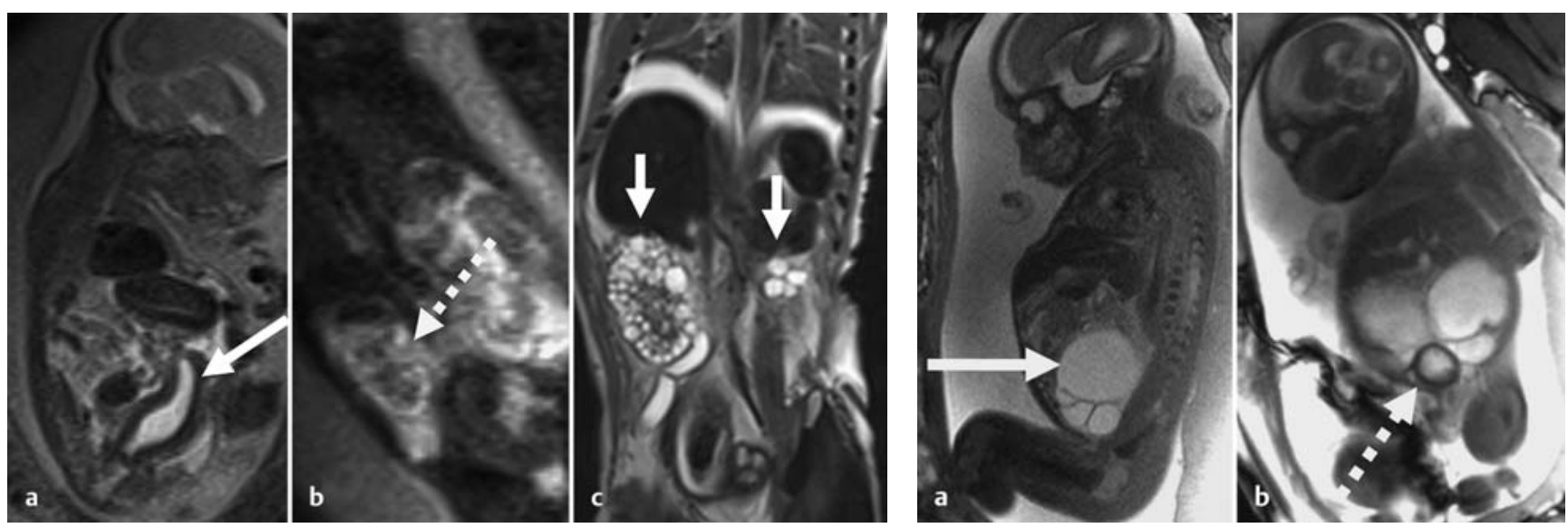

Abb. 37 a bis c Zystische Nierendysplasie und subvesikale Obstruktion, $\mathbf{a}$ und $\mathbf{b}$ pränatales MRT, c virtuelle Autopsie. a Deutlich vergrößerte Harnblase mit verdickter Wand. b Kleinzystische Veränderungen der rechten Niere. c Das postmortale MRT zeigt die zystische Nieren-

Abb. 38a und b Ausgeprägte Hydronephrose bds. Die deutlich verdickte Harnblasenwand (gestrichelter Pfeil, rechts) deutet auf eine subvesikale Obstruktion als Ursache der Hydronephrose hin.

Feten mit ARPCKD zeigen hyperintense, vergrößerte Nieren in der T2-Wichtung durch in den Tubuli gefangene Flüssigkeit. Erst im 3. Trimenon sind diese auch zu morphologisch erkennbaren Zysten gereift (Abb. 37).

Als Differenzialdiagnose kommen bei signalreichen Nieren im frühen Stadium auch andere angeborene Nierenerkrankungen infrage, wie die tuberöse Sklerose oder Tubulopathien. Hier entwickeln sich jedoch keine Zysten, und die Nieren sind meistens auch nicht vergrößert.

\section{Harnblasenanomalien}

Der wiederholt fehlende Nachweis einer Harnblase weist auf eine Blasenekstrophie hin. Nach Begleitfehlbildungen sollte dann immer mit dem MRT gesucht werden. Eine subvesikale Obstruktion ist insbesondere durch die verdickte Blasenwand und den begleitenden Reflux in die Ureteren zu erkennen (Abb. 38).

Eine verdickte Harnblasenwand mit Trabekulierung und vesikoureteralem Reflux tritt anderseits auch bei Fehlbildungen des Myelons und des Spinalkanals auf. Als weitere Differenzialdiagnose ist das Megazystissyndrom zu nennen, dieses weist allerdings keine verdickte trabekulierte Blasenwand auf, und die Hälfte dieser Fälle bildet sich spontan zurück [31].

\section{Kernaussagen}

Die erweiterten Möglichkeiten der morphologischen MRT-Diagnostik bei Feten gehören in das moderne Diagnostikspektrum jedes Fetalzentrums. Die in der Fetaldiagnostik verwendeten MRT-Sequenzen sind ultraschnelle T2- und T1-gewichtete Spezialprotokolle. Die fetale MR-Untersuchung erfolgt durch speziell geschultes Personal, da die Untersuchung interaktiv erfolgt, um sich der ständig wechselnden Lage des Feten anzupassen.

Der komplementäre Informationszuwachs durch das MRT beruht auf der deutlich höheren Kontrastauflösung und damit einer besseren Gewebedifferenzierung im Vergleich zum Ultraschall.

Jede MRT-Untersuchung benötigt eine vorausgehende qualifizierte Ultraschalluntersuchung und eine präzise formulierte Fragestellung, die auch mit der MR-Methode beantwortet werden kann.

In Einzelfällen können auch schlechte Schallbedingungen bei der Mutter - wie Adipositas per magna oder Oligohydramnion - eine fetale MRT-Diagnostik rechtfertigen, da diese Kriterien eine MRT-Untersuchung in ihrer Bildqualität nicht beeinträchtigen.

\section{Danksagung}

Für die langjährige fruchtbare, sachorientierte Zusammenarbeit bedanken wir uns bei unseren beiden engsten gynäkologischen Kooperationspartnern, Prof. H. Stepan, Leipzig, und Prof. R. Faber, Leipzig. 


\section{Über die Autoren}

\section{Franz Wolfgang Hirsch}

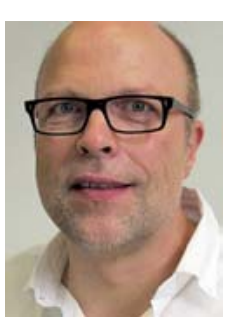

Prof. Dr. med. Studium in Halle/S. Ausbildung zum Kinderarzt an verschiedenen Kliniken. 1993/94 Arbeit am RoyalMarsden-Hospital London. Ab 1995 Ausbildung zum Facharzt für Radiologie. 1999-2002 Oberarzt an der Radiologischen Universitätsklinik Halle. 2002 Berufung als Univ.-Professor für Kinderradiologie an die Universität Leipzig. 2009 Vorsitzender der deutschen AG Kinderradiologie. 2010 Vorstandsmitglied der Gesellschaft für Pädiatrische Radiologie. 2012 Organisationskomitee Paediatric Radiology/European Congress of Radiology (Wien). 2012 Visiting Professor am Children's Hospital of Philadelphia (USA).

\section{Ina Sorge}

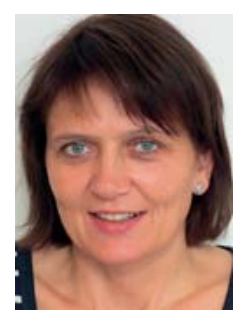

Dr. med. Studium in Leipzig. Ab 1995 Ausbildung zum Facharzt für Radiologie in der Radiologischen Praxis Telemannstraße, Leipzig, BG-Klinik Bergmannstrost, Halle, Herzzentrum Leipzig. 2003 Facharzt für Diagnostische Radiologie. Seit 2003 Abteilung für Pädiatrische Radiologie der Universität Leipzig. 2003 2006 Referenzradiologin der GPOH Morbus Hodgkin im Kindesalter. Seit 2004 leitende Oberärztin der Selbstständigen Abteilung Pädiatrische Radiologie der Universität Leipzig.

\section{Interessenkonflikt}

Prof. Dr. med. Franz Wolfgang Hirsch und OÄ Dr. med. Ina Sorge erklären, dass kein Interessenkonflikt bezüglich der Publikation „Fetale MRT-Diagnostik“ in Frauenheilkunde up2date vorliegt.

\section{Korrespondenzadresse}

Prof. Dr. med. Franz Wolfgang Hirsch

Selbstständige Abteilung für Pädiatrische Radiologie

Department für Bildgebung und Strahlentherapie

der Universität Leipzig

Liebigstraße 20a

04107 Leipzig

E-Mail: hirw@medizin.uni-leipzig.de 


\section{Literatur}

1 Weston MJ. Magnetic resonance imaging in fetal medicine: a pictorial review of current and developing indications. Postgrad Med J 2010; 86: 41 -51

2 Girard NJ. Magnetic resonance imaging of fetal developmental anomalies. Top Magn Reson Imaging 2011; 22: 11 -23

3 Kul S, Korkmaz HA, Cansu A et al. Contribution of MRI to ultrasound in the diagnosis of fetal anomalies. J Magn Reson Imaging 2012; 35: $882-890$

4 Wright C, Sibley CP, Baker PN. The role of fetal magnetic resonance imaging. Arch Dis Child Fetal Neonatal Ed 2010; 95: F137-F141

5 Al-Mukhtar A, Kasprian G, Schmook MT et al. Diagnostic pitfalls in fetal brain MRI. Semin Perinatol 2009; 33: 251 -258

6 Prayer D, ed. Fetal MRI. Berlin, Heidelberg: Springer-Verlag; 2011

7 Weston MJ. Magnetic resonance imaging in fetal medicine: a pictorial review of current and developing indications. Postgrad Med 2010; 86: $42-51$

8 Jokhi RP, Whitby EH. Magnetic resonance imaging of the fetus. Dev Med Child Neurol 2011; 53: 18-28

9 Vazquez E, Mayolas N, Delgado I, Higueras T. Fetal neuroimaging: US and MRI. Pediatr Radiol 2009; 39 Suppl 3: 422-435

10 Glenn OA. MR imaging of the fetal brain. Pediatr Radiol 2010; 40: 68-81

11 Kostovic I, Vasung L. Insights from in vitro fetal magnetic resonance imaging of cerebral development. Semin Perinatol 2009; 33: $220-233$

12 Rutherford MA. Magnetic resonance imaging of the fetal brain. Curr Opin Obstet Gynecol 2009; 21: 180-186

13 Li Y, Sansgiri RK, Estroff JA et al. Outcome of fetuses with cerebral ventriculomegaly and septum pellucidum leaflet abnormalities. Am J Roentgenol 2011; 196: W83-92

14 Yin X, Na Q, Chen J et al. Contribution of MRI to detect further anomalies in fetal ventriculomegaly. Fetal Diagn Ther 2010; 27 : $20-24$

15 Reiss-Zimmermann M, Weber D, Sorge I et al. Heterotopie, Polymikrogyrie, Lissenzephalie und Co-Malformationen der kortikalen Hirnentwicklung. Fortschr Röntgenstr 2010; 182: 472-478

16 Bulas D. Fetal evaluation of spine dysraphism. Pediatr Radiol 2010; 40: 1029-1037

17 Herman-Sucharska I, Bekiesińska-Figatowska M, Urbanik A. Fetal central nervous system malformations on MR images. Brain Dev 2009; 31: 185-199
18 Girard N, Chaumoitre K, Chapon F et al. Fetal magnetic resonance imaging of acquired and developmental brain anomalies. Semin Perinatol 2009; 33: 234-250

19 Liu YP, Chen CP, Shih SL et al. Fetal cystic lung lesions: evaluation with magnetic mesonance imaging. Pediatr Pulmonol 2010; 45: $592-600$

20 Bulas D, Egloff AM. Fetal chest ultrasound and magnetic resonance imaging: recent advances and current clinical applications. Radiol Clin North Am 2011; 49: 805-823

21 Busin KA, Kilian AK, Schaible T et al. Reliability and validity of MR image lung volume measurement in fetuses with congenital diaphragmatic hernia and in vitro lung models. Radiology 2008; 246: $553-561$

22 Jani J, Cannie M, Peralta CFA et al. Lung volumes in fetuses with congenital diaphragmatic hernia: comparison of 3D US and MR imaging assessments. Radiology 2007; 244: 575-582

23 Jani J, Cannie M, Sonigo P et al. Value of prenatal magnetic resonance imaging in the prediction of postnatal outcome in fetuses with diaphragmatic hernia. Ultrasound Obstet Gynecol 2008; 32: $793-799$

24 Daltro P, Werner H, Gasparetto TD et al. Congenital chest malformations: a multimodality approach with emphasis on fetal MR imaging. Radiographics 2010; 30: 385-395

25 Brugger PC, Prayer D. Fetal abdominal magnetic resonance imaging. Eur J Radiol 2006; 57: 278-293

26 Huisman TA, Kellenberger C]. MR imaging characteristics of the normal fetal gastrointestinal tract and abdomen. Eur ] Radiol 2008; 65: 170-181

27 Gupta P, Sharma R, Kumar S et al. Role of MRI in fetal abdominal cystic masses detected on prenatal sonography. Arch Gynecol Obstet 2010; 281: 519-526

28 Garel C, Dreux S, Philippe-Chomette P et al. Contribution of fetal magnetic resonance imaging and amniotic fluid digestive enzyme assays to the evaluation of gastrointestinal tract abnormalities. Ultrasound Obstet Gynecol 2006; 28: 282-291

29 Makin E, Davenport M. Fetal and neonatal liver tumours. Early Hum Dev 2010; 86: 637-642

30 Abdelazim IA, Abdelkarzak KM, Ramy ARM, Mounib AM. Complementary roles of prenatal sonography and magnetic resonance imaging in diagnosis of fetal renal anomalies. Austr NZ J Obstet Gynaecol 2010; 50: 237-241

31 Chapman T. Fetal genitourinary imaging. Pediatric Radiology 2012; 42: 115-123 


\section{CME.thieme.de}

cME-Teilnahme

- Viel Erfolg bei lhrer CME-Teilnahme unter http://cme.thieme.de

- Diese Fortbildungseinheit ist 12 Monate online für eine CME-Teilnahme verfügbar.

- Sollten Sie Fragen zur Online-Teilnahme haben, unter

\section{CME-Fragen}

\section{$1 \quad$ Welche Aussage trifft zu?}

A Ein MRT ist prinzipiell bei einer Risikoschwangerschaft indiziert.

B Das MRT hat eine deutlich bessere räumliche Auflösung als der Ultraschall.

C Das MRT zeichnet sich durch einen dem US überlegenen Weichteilkontrast aus.

D Eine fetale MRT-Untersuchung wird zum Beginn der Untersuchung geplant und anschließend ohne weitere Berücksichtigung der Kindsbewegungen durchgeführt.

E Die MRT-Bildqualität des fetalen Hirnes wird durch die Ossifikation der fetalen Kalotte negativ beeinflusst.

Welches ist keine akzeptierte Indikation für das fetale MRT?
A Hirnfehlbildung
B fetale Tumoren
C Herzfehler
D Lungenfehlbildungen
E unklare Ultraschallbefunde trotz vorausgehender qualifizierter Ultraschalluntersuchung

\section{Welche Aussage ist richtig?}
A Die Energieeinstrahlung durch das Magnetfeld des MRT gefährdet Mutter und Kind.
B Für das MRT ist die Sedierung des Feten erforderlich.
C Die hohe Lautstärke im MRT kann zu Hörschäden des Kindes führen.
D Die Kontrastmittelgabe ist im fetalen MRT zur Weichteildifferenzierung erforderlich.
E Es gibt bisher keinen Nachweis einer Schädigung des Feten durch die Kernspintomografie.

\section{Welche Aussage trifft nicht zu?}
A Ein Balkenmangel führt zu einer Kolpozephalie.
B Eine Balkenagenesie ist im MRT an einem Hahnenkammzeichen erkennbar.
C Bei einer Balkenagenesie sollte man nach assoziierten Fehlbildungen suchen.
D Die Stierhornform der Seitenventrikel ist ein typisches Zeichen der Balkenagenesie.
E Eine Balkenagenesie geht immer mit schweren Entwicklungsstörungen einher.

\section{Welche Aussage ist falsch?}

A Die neuronale Migration und die Hirnfaltung verlaufen in engem zeitlichen Rahmen.

B Vor der 28. SSW ist im MRT eine 4-Schichtung des Hirnmantels erkennbar.

C Eine Störung der neuronalen Migration kann zu schwerwiegenden Entwicklungsstörungen führen.

D Bereits in der 18. SSW ist eine Vielzahl der Gyri ausgebildet.

E Migrationsstörungen und Heterotopien der grauen Substanz sind im MRT besser als im Ultraschall erkennbar. 


\section{Welche Aussage trifft nicht zu?}

A Die Ursache eines Hydrozephalus ist mittels MRT häufig darstellbar.

B Bei einer intrakraniellen Zyste ist die Zystengröße entscheidend für die Prognose des Kindes.

C Die diffuse Marklagerheterotopie ist immer mit einer Gyrierungsstörung verbunden.

D Eine sichere Diagnose einer Gyrierungsstörung ist erst ab der 30. SSW möglich.

E Die Schizenzephalie ist im MRT von einem Infarkt zu unterscheiden.

7 Was ist richtig? Eine frische Hirnparenchymblutung hat im MRT ein...
A helles Signal in T1 und dunkles in T2.
B dunkles Signal in $\mathrm{T} 1$ und $\mathrm{T} 2$.
C dunkles Signal in $\mathrm{T} 1$ und helles in $\mathrm{T} 2$.
D helles Signal in T1 und T2.
E ist gar nicht darstellbar.

$8 \quad$ Welche Aussage ist nicht richtig?

A Die zystisch adenomatoide Malformation der Lunge kommt häufig in Kombination mit einer Lungensequestration vor.

B Die Zystengröße bei der CPAM spielt keine Rolle für die Prognose des Kindes und sollte deshalb nicht bestimmt werden.

C Die absolute Lungenvolumenbestimmung bei der Zwerchfellhernie ist mittels MRT möglich.

D Eine Unterscheidung zwischen primärem Chylothorax und sekundärem Hydrothorax ist mittels MRT möglich.

E Bei einer Atresie der oberen Luftwege weisen intrapulmonale Zysten auf eine Lungenschädigung durch

Flüssigkeitsüberladung hin.

9 Welche Aussage trifft nicht zu?

A Das Kolon kann durch das helle Signal des Mekoniums in der T1-Wichtung selektiv abgebildet werden.

B Mesenchymale Hamartome sind maligne Raumforderungen der Leber, aber aufgrund ihres langsamen Wachstums ohne klinische Relevanz.

C Das MRT erlaubt eine übersichtliche Visualisierung des Bruchsackinhalts bei der Omphalozele.

D Fett lässt sich im MRT durch hohes Signal in der T1- und in der T2-Wichtung identifizieren. Teratome lassen sich daher im MRT durch ihren Fettanteil eindeutig diagnostizieren.

E Eine Dünndarmatresie oder -stenose ist an der prästenotischen Dilatation besonders gut in der T2-Wichtung erkennbar.

$10 \quad$ Welche Aussage ist falsch?

A Eine Erweiterung der Ureteren ist in der T2-Wichtung besonders gut zu visualisieren.

B Eine Erweiterung der Ureteren lässt sich in der T1-Wichtung gut visualisieren, da sich erweiterte Ureteren in der T1-Wichtung immer hell darstellen.

C Eine subvesikale Obstruktion ist durch die verdickte Blasenwand und den begleitenden Reflux in die Ureteren zu erkennen.

D Die autosomal-rezessive polyzystische Nierenerkrankung (ARPCKD) zeigt beidseits hyperintense, vergrößerte Nieren in der T2-Wichtung.

E Beim Megazystissyndrom ist die Blasenwand nicht trabekuliert und nicht verdickt. 